\title{
Dimethyl Fumarate Abrogates Growth and Metastasis of Hepatocellular Carcinoma by Regulating NRF2/Bcl-XL Axis and Synergizes with Sorafenib
}

\author{
Oluwasijibomi Damola Faleti \\ Southern Medical University \\ Mingjiao Zhang \\ Southern Medical University \\ Haiqi Tan \\ Southern Medical University \\ Yibing Gong \\ Southern Medical University
}

Yuanbin Zhang

Southern Medical University

\section{Simin Deng}

Southern Medical University

Pengfei Liu

Southern Medical University

Jing Huang

Southern Medical University

\section{Gongfa Wu}

Guangzhou Medical University

\section{Xiong Wang}

City University of Hong Kong

\section{Ying Chen}

City University of Hong Kong

Mingliang He

City University of Hong Kong

Xiaoming Lyu ( $\square$ lvxiaoming6108@126.com)

The Third Affiliated Hospital of Southern Medical University https://orcid.org/0000-0002-6243-3536

\section{Research}


Keywords: Dimethyl fumarate, hepatocellular carcinoma, endoplasmic reticulum stress, B-cell lymphomaextra large $(\mathrm{Bcl}-\mathrm{xL})$, chemoresistance

Posted Date: November 16th, 2021

DOI: https://doi.org/10.21203/rs.3.rs-1045205/v1

License: (c) (1) This work is licensed under a Creative Commons Attribution 4.0 International License. Read Full License 


\section{Abstract}

\section{Background}

Emerging evidence indicates that the mitochondria play an important role in poor treatment outcomes observed in liver malignancies. Hepatocellular carcinoma (HCC) remains a leading cause of cancer mortality worldwide, and there is still no effective chemotherapeutic treatment. Dimethyl fumarate (DMF), a derivative of fumaric acid, is used to treat psoriasis and multiple sclerosis.

\section{Methods}

HCC mice model was used in this study. Eight-week-old mice bearing tumor were administered either DMF $(30 \mathrm{mg} / \mathrm{kg} / \mathrm{day} ; \mathrm{n}=6)$ or DMSO (control group; $\mathrm{n}=6)$ for 2 weeks. RNA-seq analysis was carried out on DMF-treated cells, and validation was done in two different HCC cell lines. Changes in subcellular structures were analyzed with electron microscopy. The expression of Bcl-xL, Nrf2, p-STAT3 and p-AKT were evaluated by immunoblotting.

\section{Results}

Tumour growth of HCC was significantly lower in the DMF group compared to the control group (605.84 \pm $65.06 \mathrm{~mm} 3$ and $232.1 \pm 57.14 \mathrm{~mm} 3, \mathrm{p}<0.01)$. Molecular and morphological studies revealed significant perturbation in the mitochondria accompanied by disruption of the NRF2/Bcl-xL axis. Enforced Bcl-xL expression in HCC cells markedly reversed DMF-induced apoptosis and induction of mesenchymal-toepithelial transition. Notably, we also identified the activation of unfolded protein response as a potential marker for poor response. More strikingly, DMF synergistically enhanced sorafenib's antitumor effect both in vitro, in zebrafish and mice xenograft models.

\section{Conclusion}

Collectively, our results demonstrate new mechanism insights into the antitumor effects of DMF and support the design of novel therapeutic options for HCC treatment.

\section{Background}

As the most common liver malignancy, hepatocellular carcinoma ( $\mathrm{HCC})$ is one of the most aggressive cancers worldwide, with few therapeutic options at advanced stages. About 841,000 new HCC cases and 782,000 deaths were recorded in 2018 [1, 2]. The incidence is projected to increase owing to the prevalence of metabolic disorders and viral infections $[3,4]$. The current treatment regimen is palliative and unsatisfactory. Administration of sorafenib extends patients' median overall survival by 3 months with strong side-effects and drug toxicity, while only $10-37 \%$ of patients are eligible for surgical resection $[5,6]$. The unmet clinical needs have led to the search for new strategies to improve treatment response in HCC. 
Apoptosis initiation, regulated by the delicate balance of $\mathrm{Bcl}-2$ family proteins, is frequently disrupted in $\mathrm{HCC}$. As a consequence, these apoptotic regulators constitute attractive therapeutic targets. Amongst the $\mathrm{Bcl}-2$ family proteins, B-cell lymphoma-extra large (Bcl-xL), is highly expressed in one-third of HCC patients and contributes to therapeutic resistance and low survival rates in $\mathrm{HCC}[7,8]$. Dimethyl fumarate (DMF) is a derivate of fumaric acid that has demonstrated promising results in the treatment of psoriasis and multiple sclerosis treatment. There is an ongoing phase II clinical trial for Cutaneous T Cell Lymphoma (CTCL) treatment (NCT02546440). However, a comprehensive preclinical study assessing the potential of DMF for HCC treatment as a combined or single therapy is lacking.

In addressing the unmet need for effective HCC therapeutic, we provide a comprehensive evaluation of DMF's therapeutic potential on HCC. Treatment with DMF inhibited tumour growth and metastasis by targetting the NRF2-Bcl-xL axis and resulted in accumulation of damaged mitochondria. Furthermore, DMF was as efficient as sorafenib in zebrafish xenograft, and their combination synergistically improved treatment outcome. Importantly, we identified ER stress activation as a potential marker for poor response. Taken together, our study provides strong evidence to evaluate the potential of DMF, alone or in combination with sorafenib, for HCC treatment.

\section{Methods}

\section{Cell lines and cell culture}

Human HCC cell lines Huh7, HCC-LM3, and HepG2 were acquired from the American Type Culture Collection (ATCC) and maintained in Dulbecco's modified Eagle's medium supplemented with $10 \%$ fetal bovine serum (Gibco), $100 \mathrm{IU} / \mathrm{ml}$ penicillin, and $100 \mathrm{mg} / \mathrm{ml}$ streptomycin. Cells were cultured at $37^{\circ} \mathrm{C}$ in a $5 \% \mathrm{CO}_{2}$ incubator.

\section{Reagents}

Dimethyl fumarate (purity $>99 \%$ ) was purchased from Sigma (St. Louis, MO, USA) and dissolved in DMSO. GSK2606414 (PERK inhibitor), 448c (IRE-1 inhibitor), and tunicamycin were obtained from TargetMol (China).

\section{Cell survival and death assays}

Cell survivalwas determined using Cell Counting Kit-8 (CCK-8, Beyotime, Shanghai, China). Briefly, cells were treated with indicated concentrations of dimethyl fumarate for $24 \mathrm{~h}$ or $48 \mathrm{~h}$. Following treatment, the optical density of viable cells was measured at $450 \mathrm{~nm}$ in a spectrophotometer (Tecan Group Ltd, Männedorf, Switzerland). Cell viability assays were performed in triplicate.

Cell death was assessed using the Annexin V/7-AAD Apoptosis Detection kit (Yeasen, China). In brief, cells were collected and incubated with Annexin $V$ and 7-AAD at room temperature for 15 min in the dark. 
Subsequently, stained samples were analyzed using BD LSRFortessa flow cytometer according to the manufacturer's instructions. Annexin V/7-AAD assays were performed in triplicate.

Terminal deoxytransferase-mediated dUTP-biotin nick-end labeling (TUNEL-Alexa 640) assay was used to detect cell apoptosis in GFP-expressing cell lines. Cells were fixed by treatment with $4 \%$ paraformaldehyde for $20 \mathrm{~min}$. Cell permeabilization and staining procedures were performed according to the manufacturer's guidelines (Yeasen, China). After TUNEL staining, the nuclei was stained with DAPI and cells were imaged by fluorescence microscopy (IX70, Olympus, Tokyo, Japan). At least three independent experiments were performed.

\section{Edu staining assay}

Ethynyl-2-deoxyuridine incorporation assay was used to determine cell proliferation of huh7 in vitro. 5Ethynyl-2'-deoxyuridine (EdU), an analog of thymidine, is incorporated into dividing cells during DNA synthesis (Beyotime, Shanghai, China). In brief, cells were incubated with Edu $(20 \mu \mathrm{M})$ for $1 \mathrm{~h}$ after cells were treated with $150 \mu \mathrm{M}$ DMF or control medium for $24 \mathrm{~h}$. Afterwards, cells were fixed with $4 \%$ paraformaldehyde in PBS at room temperature for $15 \mathrm{mins}$. After rinsing thrice, cells were permeabilized with $0.3 \%$ Triton-X100 in PBS. The incorporated Edu was stained by incubating cells with $1 \mathrm{x}$ Apollo solution for $30 \mathrm{mins}$ at room temperature (RT) in the dark. Finally, cell nuclei were stained using $1 \mathrm{x}$ Hoechst nuclear dye staining for $30 \mathrm{~min}$ and then detected by fluorescence microscopy.

\section{RNA sequencing}

Total RNA was extracted from cells treated vehicle control or DMF for 48 hours using TRIzol reagent (Invitrogen), and RNeasy MinElute Cleanup Kit (Qiagen). After that, RNA integrity analysis was performed and the cDNA library was constructed using Illumina TruSeq RNA Sample Preparation Kit. RNA Sequencing was performed using Illumina Novaseq 6000 by Novogene (Beijing, China).

\section{Mitochondrial $0^{2-}$ Detection}

Mitochondrial $\mathrm{O}^{2-}$ levels were detected using MitoTracker ${ }^{\circledR}$ Red CM-XRos (Yeasen, China). Cells were washed in PBS and incubated with $150 \mathrm{nM}$ MitoSOXTM Red at $37 \mathrm{C}$ for $30 \mathrm{~min}$. Fluorescence was detected using a BD LSRFortessa flow cytometer according to the manufacturer's protocol. O2- levels were expressed as mean fluorescence intensity (MFI). Data were analyzed using FlowJo software (version 10.0.7r2; Treestar). Undetached cells were also stained after treatment and imaged using confocal microscopy.

\section{Mitochondrial membrane potential assay}

After treatment for $48 \mathrm{~h}$, cells were collected in $0.5 \mathrm{~mL}$ medium and incubated with $0.5 \mathrm{~mL}$ JC-1 (Yeasen, China) working solution. Cells were maintained at $37^{\circ} \mathrm{C}$ in a $5 \% \mathrm{CO}_{2}$ incubator for $30 \mathrm{~min}$. After that, the cells were centrifuged at $1000 \mathrm{r} / \mathrm{min}$ for $3 \mathrm{~min}$ at $4{ }^{\circ} \mathrm{C}$. The mitochondrial membrane potential $(\Delta \Psi \mathrm{m})$ of cells was detected with flow cytometry according to the manufacturer's instructions. 


\section{Quantitative PCR}

RT-qPCR assays were carried using a total of $1 \mu \mathrm{g}$ RNA extracted from huh7 cells. cDNA was synthesized using HiScript ${ }^{\circledR}$ III-RT SuperMix for qPCR kit (Vazyme) following the manufacturer's protocol. Real-time qPCR was performed using qPCR SYBR Green Master Mix (Q111-02, Vazyme). PCR was performed in the relative quantification of mRNA expression using the comparative cycle threshold (CT) method. 18S rRNA expression was used as the endogenous control. The sequence of primers used can be found in the supplementary file.

\section{Western blot}

Cells were washed with PBS and lysed with ice-cold lysis buffer $(50 \mathrm{mM}$ Tris-HCl, $\mathrm{pH} 7.4,150 \mathrm{mM} \mathrm{NaCl}$, $1 \%$ Triton X-100, $0.1 \%$ SDS) supplemented with a Roche protease inhibitor cocktail and phosphatase inhibitor. After centrifugation at $4^{\circ} \mathrm{C}(14,000 \mathrm{rpm})$ for $20 \mathrm{~min}$, the supernatant was collected. Protein concentrations were measured and separated by 8-12\% SDS-polyacrylamide gel electrophoresis and then blotted onto a polyvinylidene difluoride (PVDF) membrane (GE, MA, USA). Membranes were blocked with $5 \%$ bovine serum albumin (BSA) in Tris-buffered saline-Tween 20 buffer (20 mM Tris-HCl, pH 7.4, 150 $\mathrm{mM} \mathrm{NaCl}, 0.1 \%$ Tween 20 ) for $1 \mathrm{~h}$ and incubated with specific antibodies and the corresponding HRPconjugated secondary antibodies and visualized with C600 western blot imaging system from Azure Biosystems.

\section{Transmission and Scanning electron microscopy (TEM/SEM)}

Sample preparation for electron microscopy was carried out with aid from the Imaging laboratory at Guangdong Institute of Agricultural Sciences. In brief, samples for transmission electron microscopy were fixed in $2.5 \%$ glutaraldehyde for $30 \mathrm{~min}$ at room temperature and then post-fixed with $2 \%$ osmium tetroxide for $1 \mathrm{~h}$ at $4^{\circ} \mathrm{C}$. This was followed by dehydration in increasing concentration of ethanol (30\%-100\%) and polymerization by epoxy resin. Ultra-thin sections were obtained and double-stained with $4 \%$ uranyl acetate and lead citrate. Finally, the ultrastructure of the cells was observed under a Tecnai G2 F20 S-TWIN transmission electron microscope (FEI). Cellular morphology was studied using SEM. Cells were fixed in $2.5 \%$ glutaraldehyde. After washing in phosphate buffer, samples were subjected to postfixation with $1 \%$ OsO4 and dehydrated with an increasing concentration of ethanol. Subsequently, samples were dried and coated with Au-Pd (80:20) using a Polaron E5000 sputter coater. Images were then taken with the SE detector.

\section{Transfection}

A lentivirus expressing human BCL2L1 (Bcl-xL) and NFE2L2 (NRF2) plasmid was purchased from RiboBio (Guangzhou RiboBio Technology, China). HCC cell lines, huh7 and hepG2, were transduced with the lentivirus along with $5 \mu \mathrm{g} / \mathrm{mL}$ polybrene and at a multiplicity of infection of 16 , for $48 \mathrm{~h}$. Lentivirusinfected cells were screened with the increasing concentration of puromycin (Sigma Aldrich, USA) for 6 days. Finally, healthy colonies were selected and cultured in 6-well plates with a fresh culture medium. 
siRNAs oligos against human PERK and IRE1 mRNA were synthesized by HippoBIO Gene Technology (Shanghai, China). Transfection was performed using Lipofectamine 2000 (Invitrogen) following the manufacturer's protocol. Western blot assays were applied to measure the knockdown efficiency of the siRNAs. A nonspecific siRNA with no homology to the human mRNA was used as the negative control.

\section{Xenograft models}

Animal experiments were performed in compliance with the institutional guidelines and prior approval from the Animal Experimentations Ethics Committee, Southern Medical University for the care and use of experimental animals. Athymic female mice (NU/NU), 4-6 weeks of age, were subcutaneously inoculated with huh7 cells. When the tumours became palpable $(0.1-0.2 \mathrm{~cm} 3)$, the mice received an intraperitoneal injection of DMF (30 mg/kg), dissolved in 10\% DMSO daily, for 14 days. Control mice were injected with DMSO alone. The same protocol was used for the second animal study. The mice received an intraperitoneal injection of DMF (30 mg/kg), oral sorafenib (30 mg/kg), or combination for 14 days. All drug were dissolved in $0.8 \%$ methylcellulose daily in the second animal study. The growth tumour curves were determined after measuring the tumour volume using the equation $V=(L \times W 2) / 2$. After the experiment, the mice were euthanized by cervical dislocation.

FLK-EGFP transgenic zebrafish embryos were obtained by natural mating and housed in a light-controlled facility with a standard 14:10 hour light/dark photoperiod and fed twice daily. Three to four pairs of zebrafish were set up for natural mating to produce embryos. For zebrafish xenotransplantation, the new FLK-EGFP transgenic zebrafish embryos will be maintained in $0.3 \%$ phenylthiourea ((PTU), SigmaAldrich). The chorions will be anaesthetized and injected with $50 \mathrm{CM}$-Dil labelled HCC cells. The embryos were observed after injection and allowed to recover for an hour at room temperature. After that, embryos were maintained at $33^{\circ} \mathrm{C}$. for the remaining experiment.

\section{Immunofluorescence staining analyses}

After treatment, cells were fixed with 4\% paraformaldehyde, permeabilized with $0.5 \%$ Triton X-100 for 15 min, blocked with PBS buffer containing 5\% bovine serum albumin, and washed with PBS three times. The cells were incubated with primary antibody for 2 hours and followed by 1 hour-incubation with Alexa Fluor-594 conjugated anti-rabbit immunoglobulin antibody. The nuclei were stained using DAPI (4=,6diamidino-2-phenylindole) for 1 hour. The images were captured on a Zeiss laser scanning microscope (LSM 880 NLO with Airyscan; Germany).

\section{Immunohistochemical staining}

Firstly, tissues were fixed in formalin. Next, the samples were embedded in paraffin and stained with the following primary antibodies E-cadherin (dilution 1:100), activated caspase-3 (dilution 1:200), or Bcl-xL (dilution 1:100) (Cell signalling technology, USA). After 1 hour incubation at $37^{\circ} \mathrm{C}$ and corresponding HRP-labelled streptavidin solution were added for $10 \mathrm{~min}$, and then stained with diaminobenzidine (DAB).

\section{Statistical analysis}


The data are expressed as the mean $\pm S D$ for three different experiments. The data were analyzed using GraphPad Prism 5 (GraphPad Software, USA). Unpaired two-tailed Student's t-tests were performed to determine the statistical significance for paired samples. $P<0.05$ was considered significant.

\section{Results}

\section{Dimethyl fumarate (DMF) represses the tumorigenicity of hepatocellular carcinoma cells in vivo}

To investigate if DMF displays anticancer effects, eight-week-old female athymic BALB/c nude mice were engrafted with Huh7 cells (Fig. 1a). When the tumours became palpable, the mice received an intraperitoneal injection of DMF ( $30 \mathrm{mg} / \mathrm{kg}$ ) or vehicle daily. At the end of 14 days, tumours were excised and measured. As shown in Fig. 1b-d, the tumour volumes after treatment were $605.84 \pm 65.06 \mathrm{~mm} 3$ and $232.1 \pm 57.14 \mathrm{~mm} 3$ (mean \pm standard deviation) in the control and treatment groups, respectively ( $p \leq$ 0.01). Tumour weight in the treatment group significantly reduced while both groups showed similar body weight profiles (Fig. S1). We then performed immunohistochemistry staining of tumour sections. As shown in Fig. 1e, a significant decrease in proliferation marker ki67 while active caspase 3 and E-cadherin increased after DMF treatment relative to vehicle treatment. These results suggested that DMF significantly inhibits tumour growth and metastasis in vivo.

To understand the underline antitumor effects of DMF administration, analysis of transcriptional pathways was carried out on Huh7 cells treated with DMF for 48 hours. Compared with gene expression in the control cells, 2031 upregulated genes and 1891 downregulated genes were found in the cells treated with DMF ( $|\log 2 \mathrm{FC}| \geq 1$ and P-value $\leq 0.001$, Fig. 1f). Interestingly, we observed the modulation of many genes involved in DNA replication and repair, cell proliferation, metastasis, metabolism, and protein folding in DMF-treated cells (Fig. 1g, h). Next, we selected several genes in each group and evaluated their mRNA levels by RT-qPCR. Our analysis verified the altered expression of the genes in both Huh7 and HCCLM3 cells, e.g., cell cycle regulators CDC6 and MCM7 are significantly downregulated in the DMF-treated cells (Fig. 1i). The transcriptomic results suggested that DMF exerts regulatory effects on vital pathways involved in oncogenesis and tumour progression. Taken together, these data demonstrated that DMF treatment significantly inhibited tumour growth in vivo and that variety of pathways were involved in the inhibition of tumorigenicity.

\section{DMF represses cell proliferation and induces apoptosis}

Based on signaling pathways from the above bioinformatics study, we then performed in vitro experiments to confirm the antiproliferation effects of DMF on hepatocellular carcinoma using cell counting kit-8 (CCK-8) assay. Incubation of cells with DMF resulted in reduced cell viability in a time and dose-dependent manner (Fig. 2a, Fig. S2). Next, EdU incorporation assay was performed to assess cell division after DMF treatment. We observed that DMF treatment reduced the number of dividing cells (Fig. $2 b)$, which was accompanied by increased DNA damage and altered cell morphological features, as indicated by a dose-dependent and a time-dependent increase of p-H2A.X (Fig. 2c, d). 
To clarify whether DMF-induced cell growth inhibition is associated with apoptosis, annexin V/7AAD staining was performed. Obviously, DMF treatment caused a time-dependent increase of apoptotic (annexin $\mathrm{V}+$ ) cells, compared to control cells without DMF treatment (Fig. 2e, f). Next, we investigated the expression of apoptotic-related proteins, including active caspase-3 and c-PARP, by western blot assay (Fig. 2g, h). Similarly, DMF treatment induced the cleavage of apoptotic markers, including both caspase3 and PARP. Furthermore, the incubation of HCC cells with the pan-caspase inhibitor Z-VAD-FMK significantly reduced the cell death induced by DMF (Fig. 1i). In summary, DMF inhibited hepatoma cell growth in vitro by repressing cell division and activating caspase-dependent apoptosis.

\section{DMF inhibit HCC metastasis in vitro and in vivo}

Given that metastasis contributes to poor survival rate and high recurrence in $\mathrm{HCC}$, we explored the effects of DMF on metastasis. Increased cell motility, a gain of migratory and invasive abilities play an important role in cancer metastasis[9]. We examined the potential inhibitory effects of DMF on cancer metastasis using transwell migration assays. Interestingly, we observed that DMF reduced the HCC cells' migratory capacity in a dose-dependent manner (Fig. 3a, b), further supporting our earlier results. To explore its in vivo inhibitory effects on cancer metastasis, we established an HCC-zebrafish metastatic xenograft model. Dil-labelled huh7 cells were injected into the duct of Cuvier of FLK-EGFP zebrafish at 48 h post-fertilization (hpf) and then treated with either vehicle or DMF. Notably, we observed fewer metastases in the bodies of the larvae and reduced tumour size in the treatment group compared to the control, further confirming the antimetastatic potential of DMF (Fig. 3c, d).

Epithelial-to-mesenchymal transition (EMT) has been implicated in HCC metastasis and is characterized by dysregulation of mesenchymal and epithelial markers. We next tested whether DMF could block the EMT program in HCC cells. Consistently with in vivo results, DMF treatment induced the downregulation of mesenchymal markers ( $\mathrm{N}$-cadherin and $\beta$-catenin) and the upregulation of epithelial marker ( $\mathrm{E}$ cadherin) (Fig. 3e). The results were further verified using immunofluorescence analysis in HCC cells after DMF treatment (Fig. 3f). Together, our data showed that DMF inhibited the metastatic potential of HCC cells both in vitro and in vivo.

\section{DMF induces mitochondrial dysfunction and ATP depletion}

Fumarates are readily transported into the mitochondria [10]. Next, we mapped the morphological effects of DMF on various cellular organelles. Huh7 cells were treated and analyzed with transmission electron microscopy (TEM). The untreated Huh-7 cells showed intact organelles ultrastructures such as nucleus membrane, mitochondria consisting of well-defined cristae, and electron-dense mitochondrial matrix (Fig. 4a). In contrast, the DMF-treated cells exhibited the disrupted and engulfed mitochondria, swollen morphology with a visible accumulation of vacuoles in the cytosol, and severely swollen endoplasmic reticulum (ER).

The presence of disrupted mitochondria and extensive mitophagy suggested DMF-induced mitochondrial impairment. Next, we investigated the effect of DMF exposure on mitochondrial membrane potential. 
Consistently, after treatment with DMF for 48 hours, a significant loss of mitochondrial membrane potential (Fig. 4b) displayed in DMF-treated cells, correlated with the release of cytochrome c into the cytosol (Fig. 4c). As previously reported, compromised mitochondria are often associated with amplified superoxide production and ATP depletion. Therefore, we further examined the level of mitochondrial superoxide (MitoSOX) production upon DMF treatment. Our results from flow cytometric analyses showed that DMF triggered an increase in MitoSOX compared to control (Fig.4d). Similarly, we observed a marked reduction of ATP level (Fig.4e) and compensatory activation of AMPK/ACC in DMF-treated cells as compared to the control (Fig.4f, g), further suggesting that mitochondrial dysfunction and membrane integrity damage were induced by DMF exposure. Together, our data clearly demonstrated that DMF triggered extensive mitochondria stress and that mitochondria seemed to involve in DMF-induced cell death.

\section{DMF induces apoptosis and metastasis inhibition through downregulating Bcl-xL}

Cell apoptosis is mainly initiated through the extrinsic or intrinsic pathways. Mitochondria participate in the intrinsic apoptosis arm through the Bcl-2 family proteins. Our earlier results suggested that DMF induced caspase-dependent apoptosis and extensive mitochondrial stress. Next, we assessed the role of mitochondrial in DMF-induced apoptosis. The expression of several Bcl-2 family proteins was assessed using immunoblot assays. We observed an increased level of proapoptotic Bax and Bak accompanied with marked Bcl-xL downregulation (Fig.5a-c, Fig.S3a). Unlike other antiapoptotic Bcl-2 proteins, Bcl-xL is highly expressed in one-third of HCC patients and contributes to their therapeutic resistance and low survival rates[7, 8].

Based on this, we next examined whether DMF inhibits HCC progression by downregulating Bcl-xL. Firstly, we stably infected HCC cells with either empty- or Bcl-xL-expressing vectors and treated them with vehicle or DMF. TUNEL staining assay revealed that Bcl-xL-overexpression significantly reduced apoptosis rate in DMF-treated cells (Fig.5d Fig.S3b, c). Additionally, as Bcl-xL is known to regulate mitochondria integrity, we tested whether Bcl-xL overexpression could modulate the levels of cytosolic cytochrome $c$ in DMFtreated cells. Interestingly, immunoblot assay revealed that DMF-induced cytosolic cytochrome c was significantly decreased in Bcl-xL overexpressing cells (Fig. 5e). Together, these findings suggested that $\mathrm{Bcl}-\mathrm{xL}$ downregulation by DMF contributed to cytochrome $\mathrm{c}$ release, caspase activation, and ultimately apoptosis.

Next, we further validated the role of Bcl-xL downregulation in DMF's anticancer effects in vivo using the zebrafish model. To do this, we established zebrafish xenografts using HCC cells infected with Bcl-xL or control lentiviruses. Bcl-xL-overexpression significantly attenuated the inhibition of tumour growth induced by DMF treatment (Fig. 5f, g). Surprisingly, we observed that Bcl-xL overexpression in DMFtreated cells abrogated the metastasis inhibition induced by DMF (Fig. 5h), further confirming that Bcl-xL downregulation plays a vital role in DMF's mechanism of action. To decipher the molecular mechanism underlying Bcl-xL-dependent suppression of HCCmetastasis in vivo, the expression of epithelial marker $\mathrm{E}$ cadherin, mesenchymal markers such as $\mathrm{N}$-cadherin and $\beta$-catenin was analyzed by immunoblot assay. 
Compared with the control group, $\mathrm{Bcl}-\mathrm{xL}$ overexpression significantly increased the expression of $\mathrm{N}$ cadherin, $\beta$-catenin and decreased the E-cadherin expression in HCC cells(Fig.S3d,e). Similarly, immunofluorescence analysis revealed an increased $\mathrm{N}$-cadherin expression in tandem with decreased $\mathrm{E}$ cadherin expression in DMF-treated Bcl-xL overexpression cells as compared to the control cells (Fig.S3d). Together, these findings showed that Bcl-xL downregulation plays a vital role in DMF-induced tumour growth and metastasis inhibition both in vitro and in vivo.

\section{DMF downregulates Bcl-xL through NRF2 regulation}

The Bcl-xL decrease can be caused by either less production or increased protein degradation. Next, we investigated how Bcl-xL was downregulated in DMF-treated cells. Protein degradation is dependent on the proteasome or lysosomal degradation pathway. To clarify the role of proteolysis, we examined the effect of MG132 (ubiquitin-proteasome inhibitor) or the lysosome inhibitor $\mathrm{NH} 4 \mathrm{Cl}$ on DMF-induced $\mathrm{Bcl}-\mathrm{xL}$ reduction. Treatment with either $\mathrm{MG} 132$ or $\mathrm{NH} 4 \mathrm{Cl}$ could not significantly reverse DMF-induced $\mathrm{Bcl}-\mathrm{xL}$ downregulation or cell death (Fig. 6a,b). Then we examined the Bcl-xL mRNA level in DMF-treated HCC cells and observed significant downregulation (Fig. 6c, Fig.S4a). Nrf2 is a redox-sensitive transcriptional factor of $\mathrm{BCl}-\mathrm{xL}$ and has been reported as a target of DMF[11]. We carried out immunoblotting analyses and observed Nrf2 reduction in the DMF-treated cells (Fig. 6d,e). Next, we examined whether Bcl-xL downregulation induced by DMF was correlated with Nrf2 depletion by overexpressing Nrf2 in HCC cells (Fig. 6f). To do this, cells transfected with Nrf2 or control plasmid were treated with DMF and subjected to immunoblot analysis. Interestingly, the inhibitory effect of DMF on the protein expression of Bcl-xL was reversed by Nrf2 overexpression (Fig. $6 \mathrm{~g}$, h). Similarly, we observed the reduced apoptosis and metastasis inhibition in Nrf2-overexpressed HCC cells. Taken together, our results suggest that Nrf2 acts as an important upstream regulator in DMF-induced Bcl-xL downregulation.

\section{Endoplasmic reticulum (ER) stress protects from DMF-induced apoptosis}

We also observed endoplasmic reticulum (ER) with dilated/swollen lumen and accumulation of vacuoles (Fig S5a). The structural changes were accompanied by dysregulation of ER stress genes in RNA-Seq results, indicating a possible ER stress and activation of unfolded protein response (UPR). To confirm whether ER stress was induced, a western blot assay was performed to measure the levels of UPR proteins after DMF treatment. The expression of several UPR related genes, such as GRP78/BiP, CHOP, PERK and IRE-1, was significantly increased in a concentration- and time-dependent manner as compared with control (Fig. 7a). Activation of UPR is involved in both cell survival and cell death in cancer cells. Several studies suggest that UPR activation correlates with poor treatment outcomes in clinical settings and sorafenib-treated HCC cells[12-15]. We, therefore, examined how ER stress-adaptive cells respond to DMF treatment. Huh7 and HepG2 cells were maintained in low doses of ER stress inducer and then treated with DMF. Firstly, we observed that ER-adapted cells had a higher level of Nrf2 (Fig. 7b). This observation agreed with a recent study in hepatoma cells showing that persistent ER endowed cells with higher Bcl-xL/bad ratio[15]. We observed the reduced cell death, and caspase activation in ER stress adapted cells (Fig. 7c,d), suggesting that ER stress activation is a prosurvival mechanism. 
Next, we examined the effect of UPR inhibition in DMF-treated cells. We pretreated cells with GSK2606414 (PERK), 4 $\mu 8 \mathrm{c}$ (IRE-la) or vehicle for 1 hour and further treated them with vehicle or DMF for another 48 hours. Notably, we found that PERK inhibition exacerbated the cytotoxic effects of DMF as evidenced by increased apoptotic (annexin V+) cells. In contrast, IRE-la inhibition didn't significantly influence DMF-induced cell death (Fig. 7e,f). Similar results were observed in IRE1 and PERK knockdown cells Fig. 7g,h). Our result agreed with the clinical observation that the activation of the PERK signalling arm correlated with the poor prognosis of HCC. Although in vitro ER-adapted cells might not fully portray the condition in HCC patients, our results show increased Nrf2 and reduced response to DMF in ERadapted cells. Taken together, our data clearly demonstrate that DMF triggers activation of the UPR pathway as a prosurvival mechanism and that ER stress adapted cells might respond poorly to DMF treatment.

\section{DMF enhances the efficacy of sorafenib both in vitro and in vivo}

HCC exhibits remarkable intra-and inter-tumour molecular heterogeneity. This characteristic has been linked to poor treatment response and further suggests that targeting a single pathway may not be an optimal approach $[16,17]$. Next, we examined whether DMF could enhance the therapeutic effects of sorafenib. Firstly, we analyzed the cell apoptosis induced by combined sorafenib and DMF treatment in HCC cell lines. As shown in Fig. 8a, sorafenib alone at $5 \mu \mathrm{M}$ increased the percentage of Annexin Vpositive cells by $2.4 \%$. Co-treatment of Huh7 cells with sorafenib and DMF significantly increased apoptotic (annexin $\mathrm{V}+$ ) cells by $18.3 \%$ compared to DMF only. Similarly, we observed increased expression of c-PARP after co-treatment (Fig. 8b). To explore the mechanism underlying the observed synergistic effect, we examined the signal transduction of AKT and STAT3. Increased levels of p-AKT correlate positively with poor survival rates in HCC patients receiving sorafenib treatment, while STAT3 is a major target of sorafenib[18-20]. Interestingly, immunoblot assay revealed that co-treatment sorafenib with DMF decreased the expression of p-AKT and p-STAT3 compared to sorafenib alone (Fig. 8c).

To assess the in vivo efficacy of the sorafenib and DMF combination, a zebrafish xenograft model was established and randomly assigned to four groups: control, sorafenib, DMF, and the combination of sorafenib plus DMF. Notably, treatment with DMF and sorafenib markedly enhanced the inhibitory effect on tumour growth compared with the sorafenib treatment alone in the xenograft model (Fig.S6a). For further validation, we established Huh7-C57BL/ 6 xenograft. First, mice bearing HCC cells were divided into 4 groups (control, DMF, sorafenib, sorafenib plus DMF) and treated accordingly for 14 days. After completion of treatment, tumour volume in all groups significantly reduced compared with the control group. In comparison with the sorafenib group, co-treatment of DMF and sorafenib markedly decreased tumour volumes (Fig. 8d-e). There were no significant differences in body weight between the treatment groups (Fig. 8f). Furthermore, we performed hepatotoxicity and nephrotoxicity assay to the impact of the combination therapy. The co-treatment significantly lowered the levels of AST and ALT, while the sorafenib only group showed significantly lower levels of creatinine compared to the control group (Table 1). These biochemical results showed that the combination therapy did not induce renal or hepatotoxicity 
in the treated mice, demonstrating the combined treatment of DMF and sorafenib as a potential therapeutic approach with low toxicity for the treatment of HCC.

\section{Discussion}

In the present study, we provided a comprehensive preclinical assessment of DMF in HCC to date and offered evidence for its use as a single therapy or in combination with sorafenib. Ultrastructural and molecular analysis revealed that DMF regulated the NRF2-Bcl-xL axis in HCC cells, which led to cell division arrest, mitochondria dysfunction, metabolic reprogramming and ultimately, inhibition of proliferation and metastasis. Notably, DMF functioned synergistically with sorafenib in vitro and in vivo to suppress tumour growth and metastasis. We also revealed that intrinsic/acquired resistance to DMF is associated with activation of unfolded protein response and could potentially be used as a biomarker of non-response.

DMF has been demonstrated promising in the treatment of psoriasis and multiple sclerosis treatment [21-23]. A review of the literature indicated that DMF exerts multiple pharmacological effects, including anti-inflammatory[24, 25], immunomodulatory[26, 27] and antineoplastic effects[28-31] in different cell types. Despite these promising results, the mechanisms underlying the anticancer action of dimethyl fumarate are not fully understood, and the cellular targets of DMF in HCC cells remain unidentified. Firstly, we confirmed the antitumor effects of dimethyl fumarate by using mice tumour xenograft models. Fewer Ki-67-positive cells and reduced metastasis were observed in the tumour treated with dimethyl fumarate compared to the untreated group, indicating that DMF could inhibit tumour growth and progression in vivo. By combining electron microscopy, deep sequencing, and protein profile analysis, we showed that the mechanism of action of DMF centres around the mitochondria and is dependent on the downregulation of oncogenic protein, Bcl-xL. Mechanistically, DMF induced Bcl-xL-downregulation led to inhibition of metastasis, mitochondrial damage, caspase activation and, thus, reduced tumour growth and progression. One of the major functions of $\mathrm{Bcl}-\mathrm{xL}$ is to protect cells from proapoptotic signals and maintain neoplastic traits in tumour cells. As such, aberrant Bcl-xL expression has been associated with poor prognosis of HCC. We established a zebrafish xenograft model to validate Bcl-xL's role in DMF's anticancer effects. Consistently, we observed that DMF inhibited the oncogenicity of HCC in the xenograft model and that $\mathrm{Bcl}-\mathrm{xL}$ overexpression can attenuate DMF's effects on tumour cell growth and metastasis. Together, our in vivo and in vitro experimental data suggest that $\mathrm{Bcl}-\mathrm{xL}$ is important in the antineoplastic effects of DMF in HCC.In this study, we also observed unfolded protein response can modulate the cytotoxic effects of DMF. In the endoplasmic reticulum (ER) lumen, nascent proteins are properly folded, modified and translocated to other organelles/extracellular space. However, misfolded or unfolded proteins can accumulate under stressful conditions. To restore protein homeostasis, unfolded protein response is initiated through the activation of ER transmembrane sensors such as inositol-requiring kinase 1 (IRE1), protein kinase-like ER kinase (PERK), and transcription factor 6 (ATF6). Our results suggest that UPR activation is a cytoprotective mechanism. We observed upregulation of NRF2 and Bcl$x L$ in ER stressed HCC cells which led to a decrease in cytotoxic effects of DMF. In contrast, inhibition of the PERK arm of the UPR exacerbated the cytotoxic effects of DMF, whereas IRE1a inhibition did not 
change the effect of DMF. Our observation is further supported by a recent study that showed that ER stress adapted hepatoma cells have higher Bcl-xL/Bad[15]. Previous examination of clinical samples and HCC cells also found a link between activation of UPR and poor response to sorafenib [12-14]. Mechanisms proposed for UPR-dependent chemoresistance include PERK-mediated upregulation of MDR-related protein 1[32], Bcl-xL/Bad[15], detoxifying enzymes[33], which promote cell survival under stressful conditions. GRP78 and the IRE1 arm have also been reported to confer chemoresistance by regulating caspase-dependent apoptosis and STAT3 signalling, respectively[34-36]. Our results indicate that the ER stress-adaptive cells are less responsive to DMF treatment, suggesting that DMF therapy may be less effective for patients with high levels of ER stress.

The short-lived and poor therapeutic response of HCC has been partly attributed to intra-tumour and intertumour molecular heterogeneity $[16,17]$. Given this observation, the combination of sorafenib with other chemical drugs with alternated mechanisms of action could amplify its anticancer activity and improve patients' prognoses. Interestingly, DMF combined with sorafenib treatment exhibited greater antitumor efficacy than DMF or sorafenib treatment alone, as indicated by the marked inhibition of tumour growth and metastasis in the zebrafish and mice xenograft model. Importantly, treatment was tolerated in both xenograft models. Furthermore, our results showed that the co-treatment enhance the decrease of AKT and STAT3 phosphorylation, two markers linked to a good prognosis of HCC.

We also identified NRF2 as the upstream regulator of Bcl-xL transcription. NRF2 is a CNC-bZIP transcription factor that serves as the master regulator of cytoprotective and detoxification genes. The NRF2 and its downstream target genes have been implicated in drug resistance and cancer progression. Overexpression of NRF2 renders DMF-treated cells refractory to the DMF-induced cytotoxic effects, suggesting the functional relevance of NRF2 modulation (Fig. 6). This is consistent with existing literature that DMF can downregulate NRF2 in cancer cells at cytotoxic concentration[30, 37].

\section{Conclusion}

Our present study revealed important details regarding the anticancer effects of dimethyl fumarate in HCC. Mechanistically, DMF inhibited cancer cell growth and metastasis by targetting the Nrf2-Bcl-xL axis in HCC cells. Our findings support further evaluation of DMF as a single therapy or in combination with sorafenib. Activation of the UPR could be used as a predictor of resistance to DMF. Additional studies might be needed to identify the patient subgroup that will benefit from DMF treatment.

\section{Abbreviations}

HCC: heptocellular carcinoma; NRF2: nuclear factor erythroid 2-related factor 2; Bcl-xL: B-cell lymphoma extra-large; ER: endoplasmic reticulum stress; PARP-1: poly (ADP-ribose) polymerase-1; CDC6: Cell division cycle 6; MCM7: Minichromosome Maintenance Complex Component 7; SSBP1: Small acid-soluble spore protein B; XBP1: X-Box Binding Protein 1; RNF185: Ring Finger Protein 
185; FLCN: Folliculin; CARS2: Cysteinyl-TRNA Synthetase 2; LAMB1: Laminin Subunit Beta

1; MitoSOX: mitochondrial superoxide; ALT: alanine aminotransferase; AST: aspartate aminotransferase.

\section{Declarations}

\section{Acknowledgments}

We appreciate our colleagues engaging in nucleic acid screening of SARS-CoV-2. from the department of laboratory medicine, The Third Affiliated Hospital, Southern Medical University, for their works and kind help.

\section{Authors' Contributions}

$\mathrm{XL}$ and MH designed the study. OF, MZ mainly performed the experiments; , HT and YG performed part of the cell experiments; $\mathrm{YZ}$ and SD performed the animal experiments. $\mathrm{PL}, \mathrm{JH}$ and $\mathrm{YC}$ performed the animal serum biochemical assay, GW and XW did the pathology slides and IHC staining; OF and XL analyzed data, $\mathrm{XL}$ and $\mathrm{MH}$ supervised the study; $\mathrm{OF}, \mathrm{XL}$ and $\mathrm{MH}$ wrote the manuscript.

\section{Funding}

The work was partially supported by grants from the National Natural Science Foundation of China, China (No. 81502335), Guangdong Basic and Applied Basic Research Foundation, China (Nos. 2020A1515010081; 2021A1515010713). Science and Technology Program of Guangzhou, China (No. 201704020127 and No. 201804010043); The Science Technology and Innovation Committee of Shenzhen Municipality [JCYJ20180507181627057]; RGC General Research Fund of Hong Kong Special Administrative Region [11104020], Strategic funds from City University of Hong Kong and Hong KongChina postdoctoral fellowship grant.

\section{Availability of data and materials}

The datasets used and/or analyzed during the current study are available from the corresponding author on reasonable request.

\section{Ethics approval and consent to participate}

All animal experiments were performed in compliance with the institutional guidelines and prior approval from the Animal Experimentations Ethics Committee, Southern Medical University for the care and use of experimental animals.

\section{Consent for publication}

All authors agreed on the manuscript.

\section{Competing interests}


The authors declare no conflict of interest.

\section{Author details}

${ }^{1}$ Department of Laboratory Medicine, The Third Affiliated Hospital, Southern Medical University. Guangzhou, China.

Oluwasijibomi Damola Faleti ${ }^{1}{ }^{2}$, Mingjiao Zhang ${ }^{1 \#}$, Haiqi Tan ${ }^{1}$, Yibing Gong ${ }^{1}$, Yuanbin Zhang ${ }^{1}$, Simin Deng ${ }^{1}$, Pengfei Liu ${ }^{1}$, Jing Huang ${ }^{1}$, Xiaoming Lyu ${ }^{1}$

${ }^{2}$ Department of Biomedical Sciences, City University of Hong Kong, HKSAR, China Oluwasijibomi Damola Faleti ${ }^{1,2}$, Xiong Wang ${ }^{2}$, Ying Chen ${ }^{2}$, Ming-Liang $\mathrm{HE}^{2,4}$

${ }^{3}$ Department of pathology, The Fourth Affiliated Hospital of Guangzhou Medical University, Guangzhou, China.

Gongfa $\mathrm{Wu}^{3}$

${ }^{4}$ Biotechology Center, CityU Shenzhen Research Institute, Nanshan, Shenzhen, China Mingliang $\mathrm{He}^{2,4}$

\section{Corresponding author}

Correspondence to Xiaoming Lyu or Mingliang He.

\section{References}

1. Forner, A., M. Reig, and J. Bruix, Hepatocellular carcinoma. Lancet, 2018. 391(10127): p. 1301-1314.

2. Bray, F., et al., Global cancer statistics 2018: GLOBOCAN estimates of incidence and mortality worldwide for 36 cancers in 185 countries. CA Cancer J Clin, 2018. 68(6): p. 394-424.

3. Liu, Z., et al., Global incidence trends in primary liver cancer by age at diagnosis, sex, region, and etiology, 1990-2017. Cancer, 2020. 126(10): p. 2267-2278.

4. Petrick, J.L., et al., International trends in hepatocellular carcinoma incidence, 1978-2012. Int J Cancer, 2020. 147(2): p. 317-330.

5. Pang, T.C. and V.W. Lam, Surgical management of hepatocellular carcinoma. World J Hepatol, 2015. 7(2): p. 245-52.

6. Llovet, J.M., et al., Sorafenib in advanced hepatocellular carcinoma. N Engl J Med, 2008. 359(4): p. 378-90. 
7. Takehara, T., et al., Expression and role of BCl-xL in human hepatocellular carcinomas. Hepatology, 2001. 34(1): p. 55-61.

8. Watanabe, J., et al., Prognostic significance of Bcl-xL in human hepatocellular carcinoma. Surgery, 2004. 135(6): p. 604-12.

9. Chaffer, C.L. and R.A. Weinberg, A perspective on cancer cell metastasis. Science, 2011. 331(6024): p. 1559-64.

10. Passarella, S., et al., Anion transport in rat brain mitochondria: fumarate uptake via the dicarboxylate carrier. Neurochem Res, 1987. 12(3): p. 255-64.

11. Niture, S.K. and A.K. Jaiswal, Nrf2-induced antiapoptotic BCl-xL protein enhances cell survival and drug resistance. Free Radic Biol Med, 2013. 57: p. 119-31.

12. Feng, Y.H., et al., Proteomic Profile of Sorafenib Resistance in Hepatocellular Carcinoma; GRP78 Expression Is Associated With Inferior Response to Sorafenib. Cancer Genomics Proteomics, 2019. 16(6): p. 569-576.

13. Won, J.K., et al., Protein disulfide isomerase inhibition synergistically enhances the efficacy of sorafenib for hepatocellular carcinoma. Hepatology, 2017. 66(3): p. 855-868.

14. Chiou, J.-F., et al., Glucose-regulated protein 78 is a novel contributor to acquisition of resistance to sorafenib in hepatocellular carcinoma. Annals of surgical oncology, 2010. 17(2): p. 603-612.

15. Wu, M.-Z., et al., LnCRNA GOLGA2P10 is induced by PERK/ATF4/CHOP signaling and protects tumor cells from ER stress-induced apoptosis by regulating Bcl-2 family members. Cell death \& disease, 2020. 11(4): p. 1-12.

16. Liu, J., H. Dang, and X.W. Wang, The significance of intertumor and intratumor heterogeneity in liver cancer. Exp Mol Med, 2018. 50(1): p. e416.

17. Nguyen, P.H.D., et al., Intratumoural immune heterogeneity as a hallmark of tumour evolution and progression in hepatocellular carcinoma. Nat Commun, 2021. 12(1): p. 227.

18. Kunter, l., et al., Active form of AKT controls cell proliferation and response to apoptosis in hepatocellular carcinoma. Oncol Rep, 2014. 31(2): p. 573-80.

19. Gu, F.M., et al., Sorafenib inhibits growth and metastasis of hepatocellular carcinoma by blocking STAT3. World J Gastroenterol, 2011. 17(34): p. 3922-32.

20. Tai, W.-T., et al., Signal transducer and activator of transcription 3 is a major kinase-independent target of sorafenib in hepatocellular carcinoma. Journal of hepatology, 2011. 55(5): p. 1041-1048.

21. Mrowietz, U. and K. Asadullah, Dimethylfumarate for psoriasis: more than a dietary curiosity. Trends in Molecular Medicine, 2005. 11(1): p. 43-48.

22. Mills, E.A., et al., Emerging Understanding of the Mechanism of Action for Dimethyl Fumarate in the Treatment of Multiple Sclerosis. Front Neurol, 2018. 9: p. 5.

23. Xu, Z., et al., Dimethyl fumarate for multiple sclerosis. Cochrane Database of Systematic Reviews, 2015(4). 
24. Lin, S.X., et al., The anti-inflammatory effects of dimethyl fumarate in astrocytes involve glutathione and haem oxygenase-1. ASN Neuro, 2011. 3(2).

25. Li, R., et al., Dimethyl Fumarate Treatment Mediates an Anti-Inflammatory Shift in B Cell Subsets of Patients with Multiple Sclerosis. J Immunol, 2017. 198(2): p. 691-698.

26. Albrecht, P., et al., Effects of dimethyl fumarate on neuroprotection and immunomodulation. $\mathrm{J}$ Neuroinflammation, 2012. 9: p. 163.

27. Bombeiro, A.L., et al., Immunomodulation by dimethyl fumarate treatment improves mouse sciatic nerve regeneration. Brain Res Bull, 2020. 160: p. 24-32.

28. Kastrati, I., et al., Dimethyl Fumarate Inhibits the Nuclear Factor B Pathway in Breast Cancer Cells by Covalent Modification of p65 Protein. Journal of Biological Chemistry, 2016. 291(7): p. 3639-3647.

29. Booth, L., et al., Regulation of dimethyl-fumarate toxicity by proteasome inhibitors. Cancer Biol Ther, 2014. 15(12): p. 1646-57.

30. Saidu, N.E.B., et al., Dimethyl fumarate, a two-edged drug: Current status and future directions. Med Res Rev, 2019. 39(5): p. 1923-1952.

31. Kastrati, I., et al., Dimethyl fumarate impairs breast cancer growth and inhibits the nuclear factor kappa B pathway in breast cancer cells by covalent modification of p65. Cancer Research, 2016. 76.

32. Salaroglio, I.C., et al., PERK induces resistance to cell death elicited by endoplasmic reticulum stress and chemotherapy. Mol Cancer, 2017. 16(1): p. 91.

33. Rouschop, K.M., et al., PERK/elF2 alpha signaling protects therapy resistant hypoxic cells through induction of glutathione synthesis and protection against ROS. Proceedings of the National Academy of Sciences of the United States of America, 2013. 110(12): p. 4622-4627.

34. Reddy, R.K., et al., Endoplasmic reticulum chaperone protein GRP78 protects cells from apoptosis induced by topoisomerase inhibitors: role of ATP binding site in suppression of caspase-7 activation. J Biol Chem, 2003. 278(23): p. 20915-24.

35. Fu, Y., J. Li, and A.S. Lee, GRP78/BiP inhibits endoplasmic reticulum BIK and protects human breast cancer cells against estrogen starvation-induced apoptosis. Cancer Res, 2007. 67(8): p. 3734-40.

36. Logue, S.E., et al., Inhibition of IRE1 RNase activity modulates the tumor cell secretome and enhances response to chemotherapy. Nat Commun, 2018. 9(1): p. 3267.

37. Zhang, Y., et al., Computational repositioning of dimethyl fumarate for treating alcoholic liver disease. Cell Death Dis, 2020. 11(8): p. 641.

\section{Tables}

Table 1 Renal and hepatic function tests were used to assess the toxicity of DMF and sorafenib and treatment. 


\begin{tabular}{|lllll|}
\hline & Control & DMF & Sorafenib & Sorafenib + DMF \\
\hline ALT $(\mathrm{U} / \mathrm{L})$ & $47.25 \pm 4.87$ & $49 \pm 4.06$ & $38 \pm 4.74$ & $31.25 \pm 3.34^{\star}$ \\
\hline AST $(\mathrm{U} / \mathrm{L})$ & $100 \pm 10.56$ & $110.75 \pm 3.27$ & $112 \pm 14.3$ & $81 \pm 4.95^{\star}$ \\
\hline Blood Urea Nitrogen $(\mathrm{mmol} / \mathrm{L})$ & $6.92 \pm 2.21$ & $8.17 \pm 1.21$ & $6.3 \pm 0.53$ & $6.15 \pm 0.18$ \\
\hline Creatinine $(\mu \mathrm{mol} / \mathrm{dL})$ & $9.25 \pm 1.79$ & $9 \pm 0.71$ & $6 \pm 0.71 *$ & $9.75 \pm 0.83$ \\
\hline Weight $(\mathrm{g})$ & $25.1 \pm 0.21$ & $24.5 \pm 0.15$ & $24.8 \pm 0.15$ & $24.5 \pm 0.17$ \\
\hline
\end{tabular}

ALT Alanine transaminase, AST Aspartate transaminase. Unpaired t-test was used for statistical analysis. * $P<.05$ Data are presented as mean $\pm S D(n \geq 3)$.

\section{Figures}


a

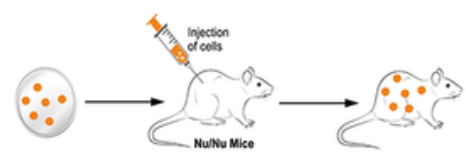

b

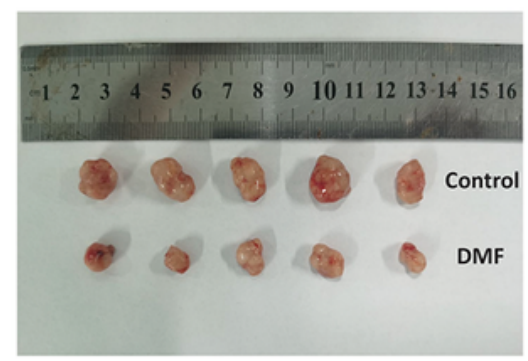

g

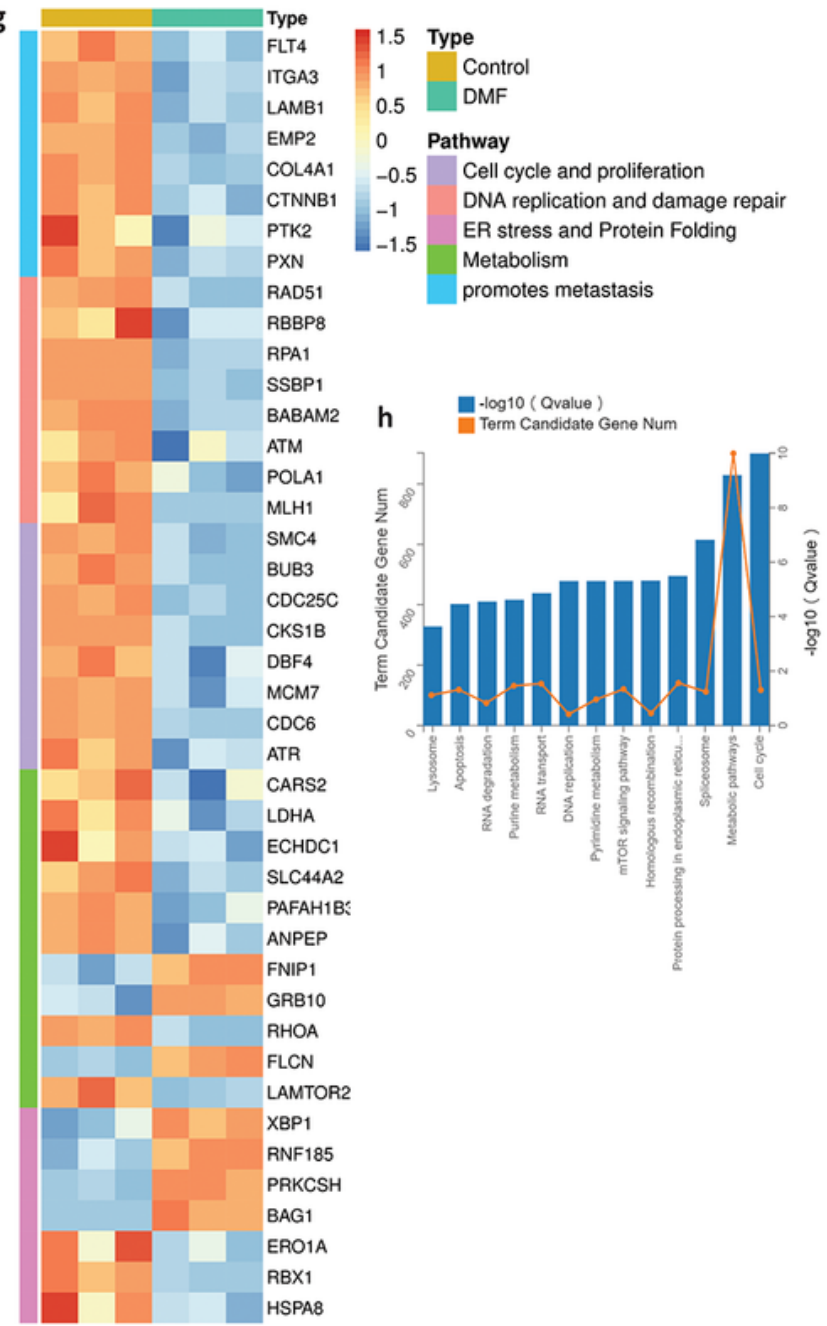

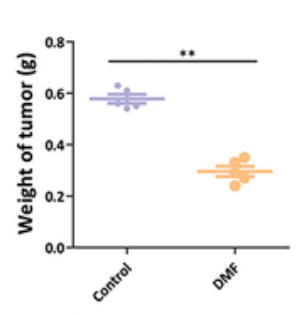
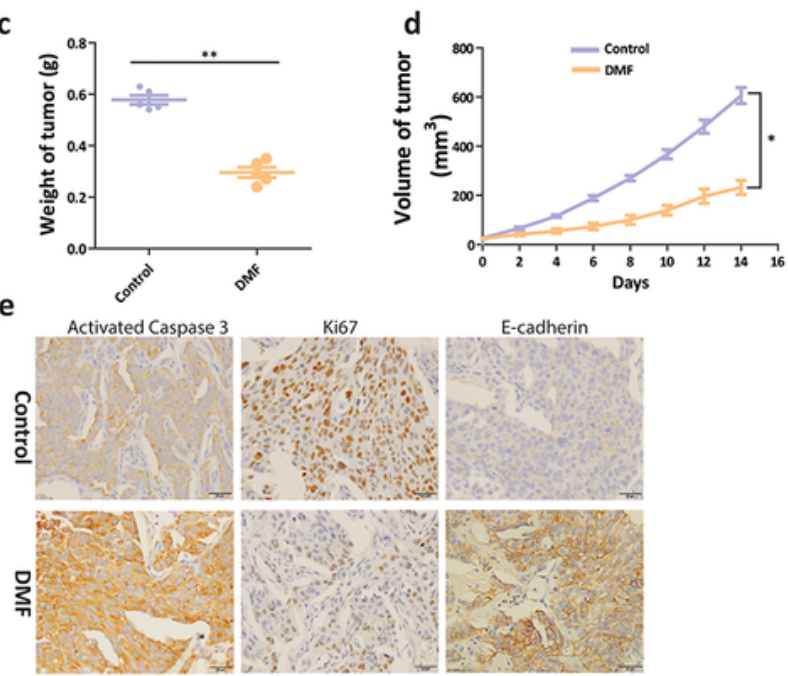

f

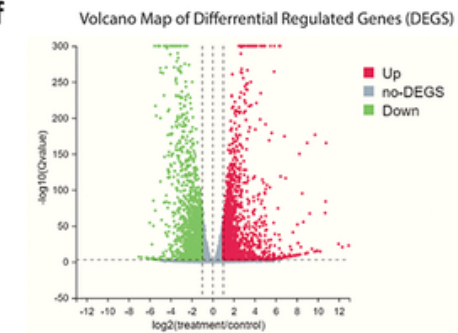

i
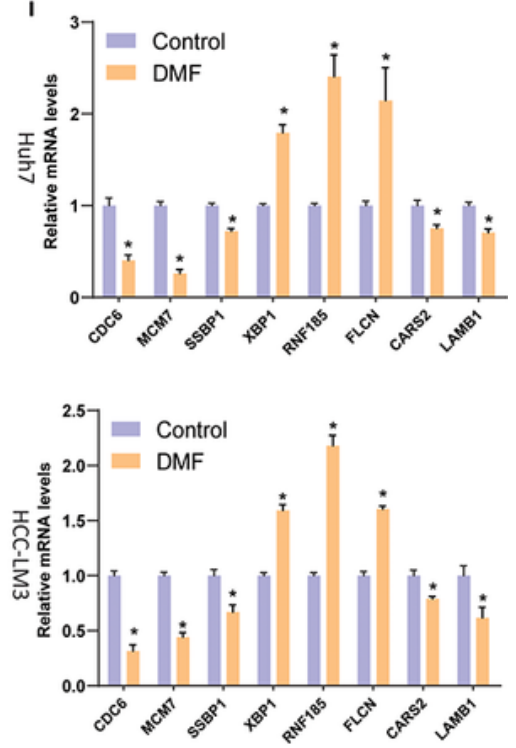

Figure 1

Dimethyl fumarate represses the tumorigenicity of hepatocellular carcinoma cells in vivo (a) A schematic of the Huh7 xenograft tumor model exposed to DMF treatment for 14 days (b) Huh7 cells were injected into the animal to establish the xenograft model. Control group mice were treated with DMSO via intraperitoneal injection, and treatment group mice were treated with DMF $(30 \mathrm{mg} / \mathrm{kg}$ body weight i.p.) daily for 14 days. (c,d) Volume and weight of the tumors in the two groups were recorded. Results are 
mean $\pm S D(n=5),{ }^{\star} P \leq 0.01$. (e) The expression levels of Ki67, E-cadherin and cleaved-caspase 3 in control and DMF treatment groups of mice were detected by immunohistochemistry. Bar scale $20 \mu \mathrm{m}$ (f) Volcano plot showing differentially regulated genes in control vehicle and DMF treated cells. (g) Heatmap of the mRNA gene expression of genes in Huh7 cells treated with DMF and vehicle for 48 hours $(\mathrm{h})$ KEGG signaling pathway analysis of differentially expressed genes in huh7-treated cells (i) qPCR analysis of transcription levels of selected genes in huh7 and HCC-LM3 cells. ${ }^{*} P<.05, * \star P<0.01$, Data are presented as mean $\pm \operatorname{SD}(n \geq 3)$.
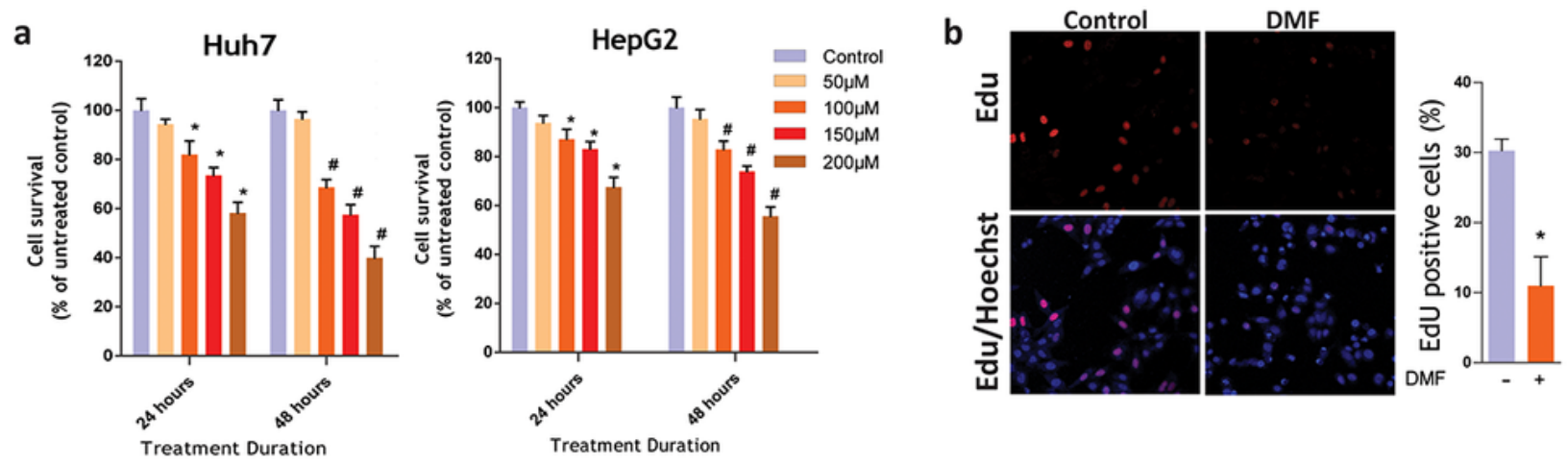

c
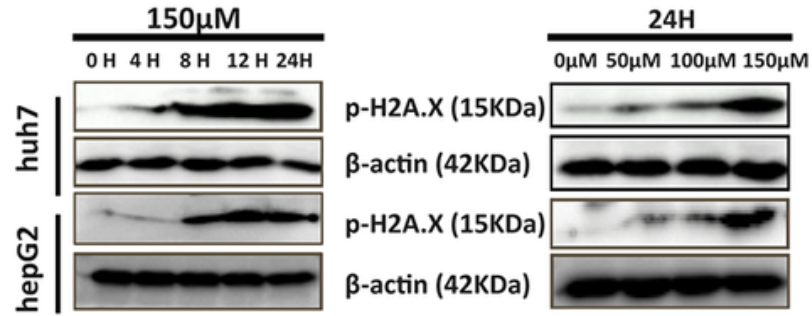

d
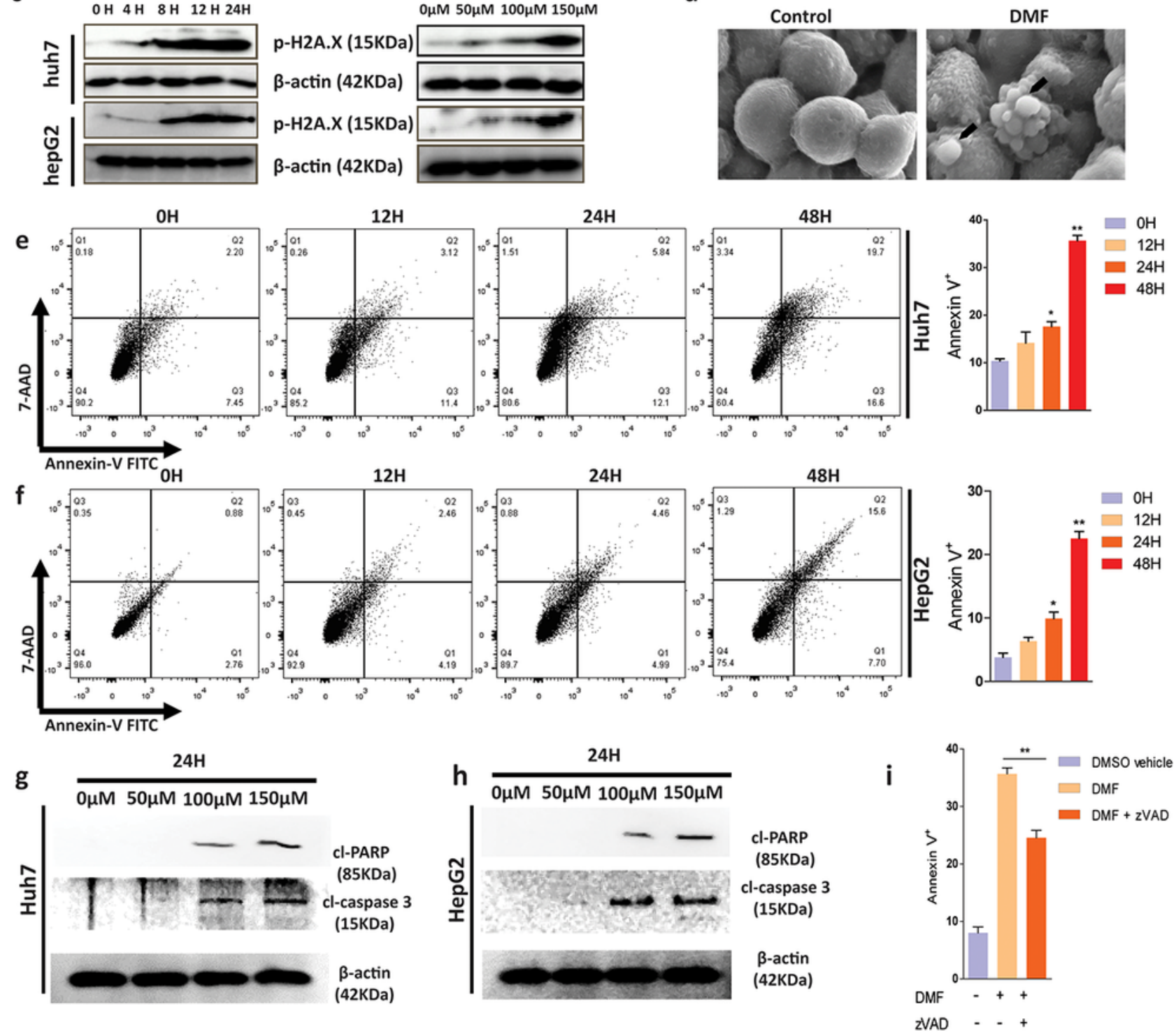


\section{Figure 2}

Dimethyl fumarate represses cell proliferation and induces apoptosis (a) Huh7 and hepG2 hepatocellular carcinoma cell lines were treated with different concentrations of DMF for 24 and $48 \mathrm{~h}$. Cell survival was measured by CCK-8 assay. (b) EdU staining was used to assess the effect of DMF on the proliferation of HCC cells. Cells were exposed to DMF $(150 \mu \mathrm{M})$ and stained using EdU assay. The percentage of EdUpositive cells (c) Western blots of DNA damage marker, p-H2A.X, in control, and DMF treated cells (d) Scanning electron microscopy images of Huh7 cells treated with DMF or DMSO. Control cells displaying normal cellular membrane while DMF-treated cells had an altered membrane structure after 24 hours of treatment $(e, f)$ Huh7 and HepG2 cells were treated with DMF in a time-dependent manner. Cell death was analyzed using Annexin V-FITC/7-AAD staining. $(\mathrm{g}, \mathrm{h})$ Western blotting analyses of cleaved-caspase3 and cleaved PARP in DMF-treated huh7 cells. (I) Huh7 cells were treated with DMF $(150 \mu \mathrm{M})$ with or without ZVAD-MFK, and cell viability was performed using CCK-8 assay. Unpaired t-test was used for the statistical analysis. *, \#P<.05, ** $\mathrm{P}<0.01$, Data are presented as mean $\pm S D(n \geq 3)$. 

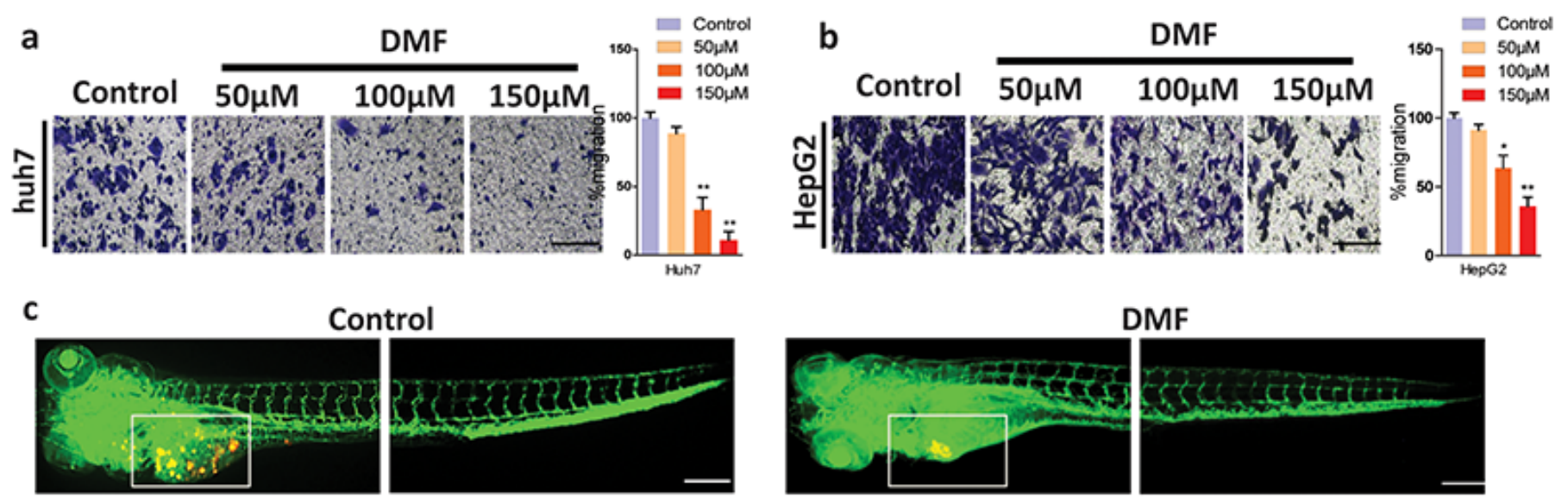

Control
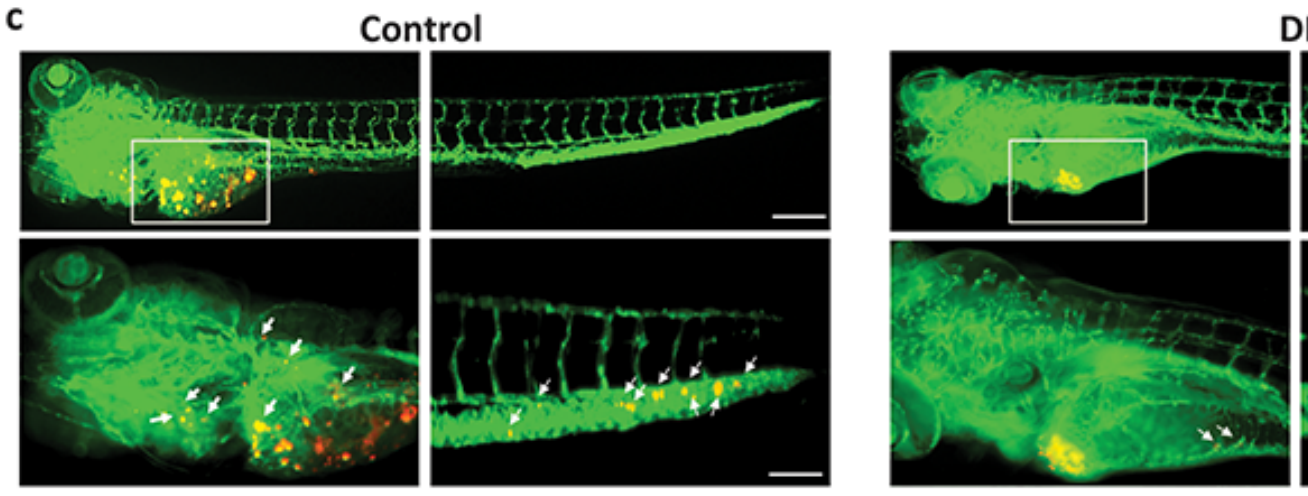

DMF
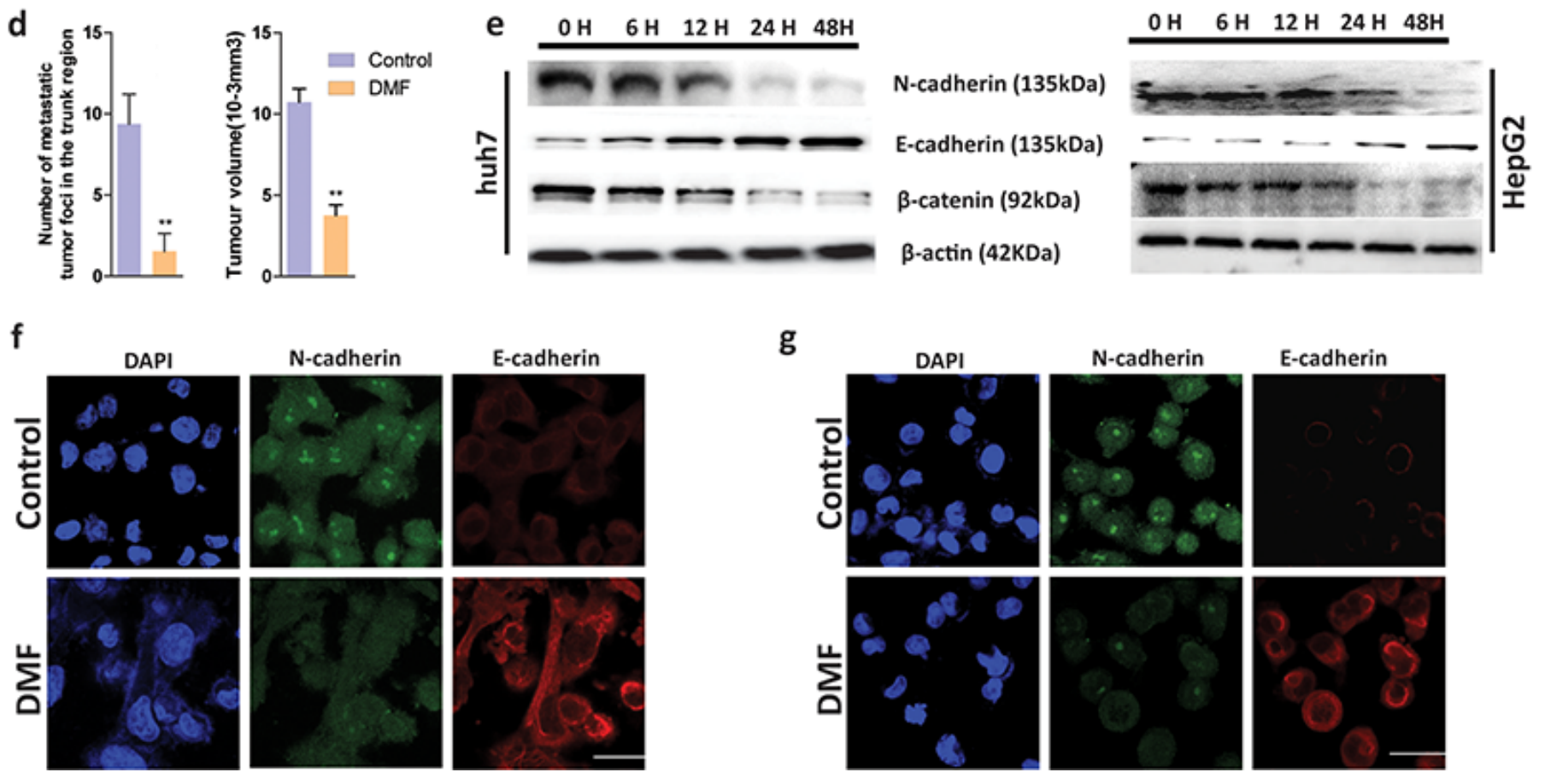

Figure 3

Dimethyl fumarate inhibit HCC metastasis in vitro and in vivo $(a, b)$ The migratory abilities of huh7 cells treated with DMF were measured using transwell assays Scale bar, $100 \mu \mathrm{m}$ (c) Representative images of head, and tail regions showing tumor cells and disseminated tumor foci in tumor-bearing zebrafish embryos treated $\operatorname{DMF}(0.5 \mu \mathrm{M})$, or vehicle control ( $n=10$ embryos/group). Arrowheads point to tumor cells. Scale bar $50 \mu \mathrm{m}$. (d) Quantification of the number of disseminated tumor foci in the trunk region and tumor volume (e) The expression of E-cadherin and N-cadherin in huh7 and hepG2 cells, in the absence or presence of $150 \mu \mathrm{M}$ DMF for 24 hours (f) Immunofluorescence staining for E-cadherin and Ncadherin in huh-7 and hepG2 cells treated with DMF or control vehicle for $48 \mathrm{~h}$. Scale bar, $20 \mu \mathrm{m} .{ }^{*} \mathrm{P}<$ 0.05 , ** $P<0.01$, Data are presented as mean $\pm S D(n \geq 3)$. 
a Veh
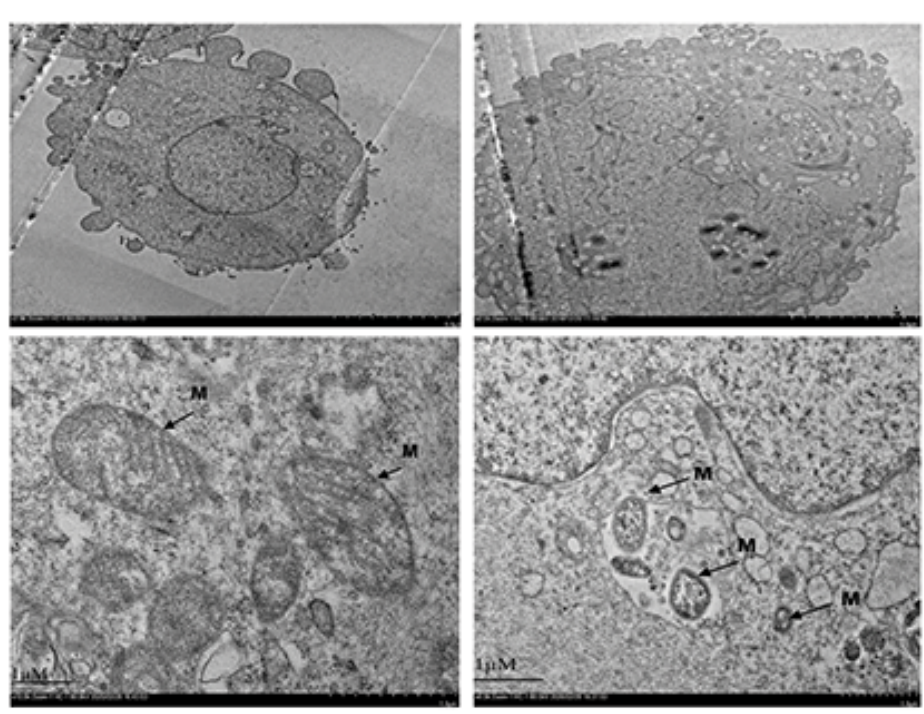

b
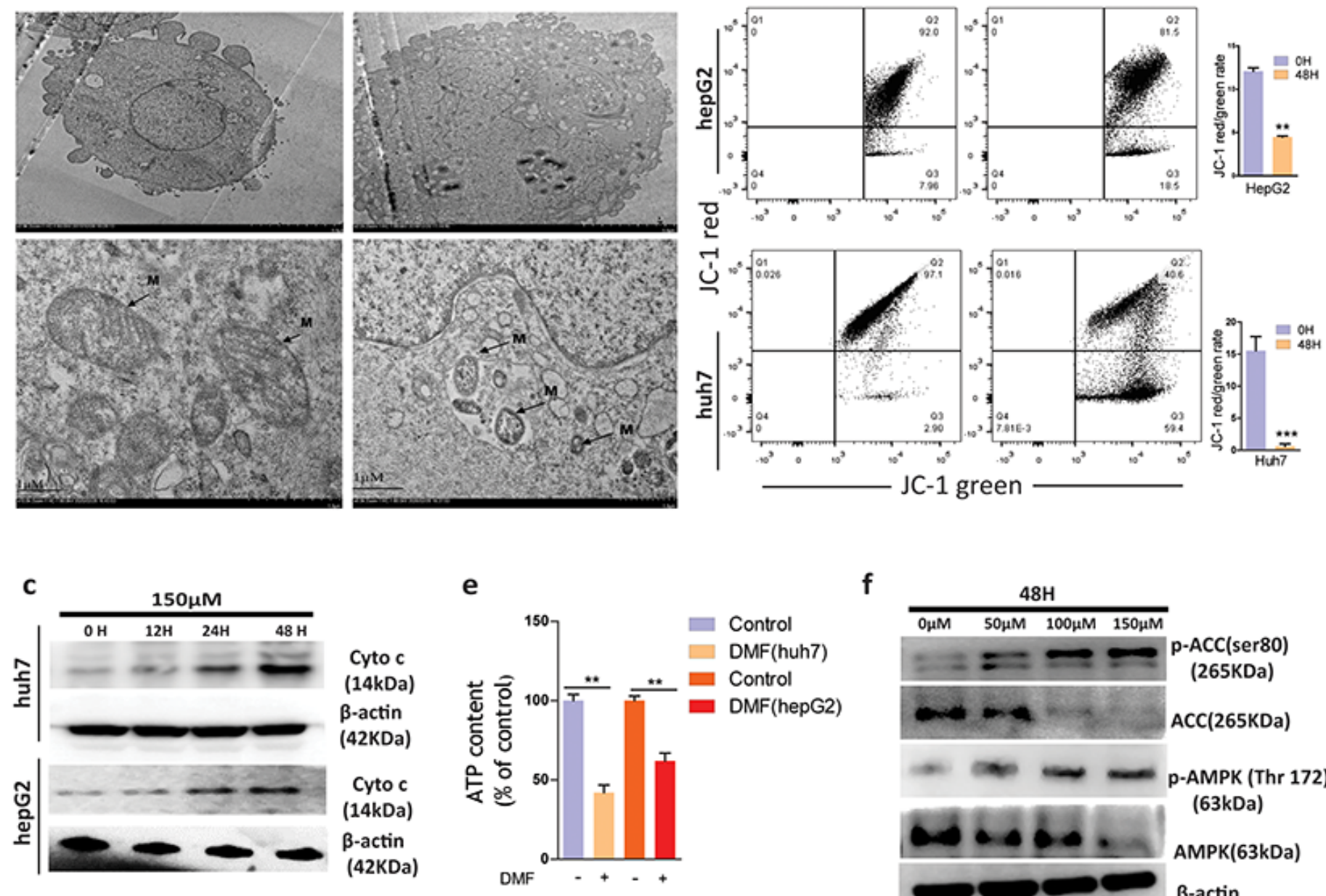

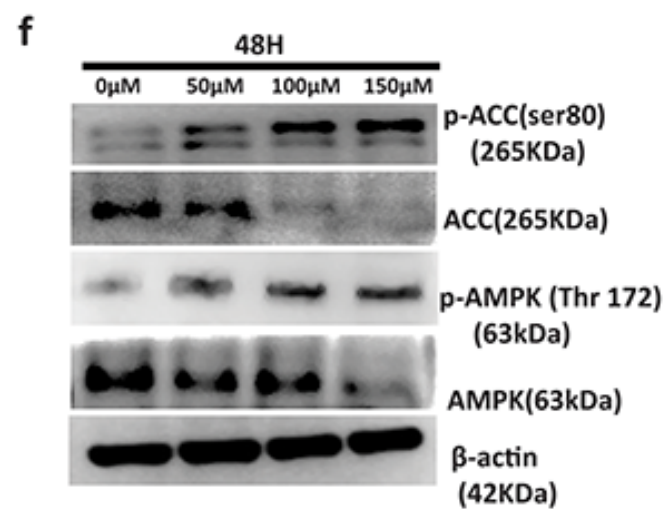

(42KDa)
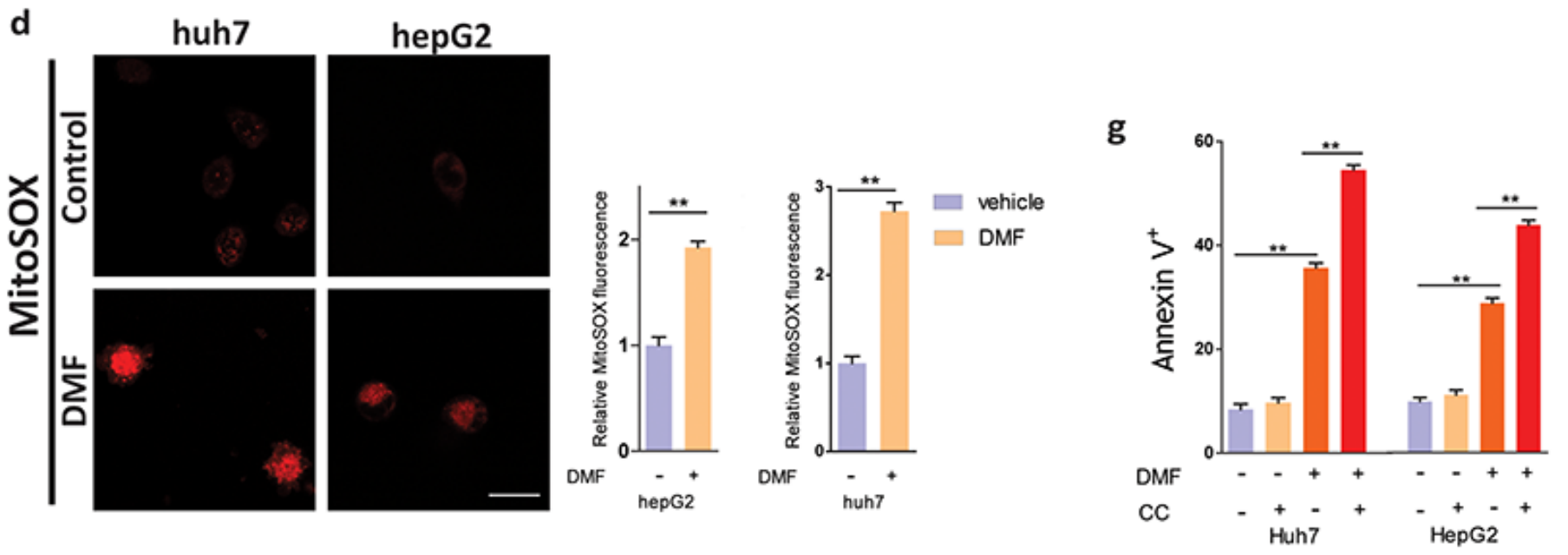

Figure 4

Dimethyl fumarate induces mitochondrial dysfunction and ATP depletion (a) Ultrastructural analysis of huh7 cells treated with DMF. Representative electron microscopic images of huh7 cells treated with DMF or vehicle control. Electron micrograph showing cristae and electron-dense mitochondrial matrix in vehicle-treated cells. Damage mitochondria were observed after DMF treatment (b) JC-1 staining by flow cytometry was used to assess mitochondrial membrane potential (MMP) after DMF treatment. 
Histograms indicate the red/green ratio of JC-1 fluorescence (c) Western blot analysis of cytoplasmic cytochrome c level in control and DMF-treated group (d) MitoSOX fluorescence images of HCC cells with or without DMF treatment mtROS level was measured by staining with mitoSOx (150 nM) for $0.5 \mathrm{~h}$. Flow cytometry results were calculated and represented as the relative value to control ( $\left.{ }^{*} p<0.05\right)$. (e) Cellular ATP levels were measured in huh7 treated with DMF or vehicle for 48 hours $(f)$ Western blot analysis of AMPK, P-AMPK, ACC, P-ACC level in DMF or vehicle treated cells $(\mathrm{g})$ Cells were treated with DMF $(150 \mu \mathrm{M})$ with or without AMPK inhibitor (compound c) and cell death was performed using Annexin V-FITC/7-AAD staining. Scale bar, $20 \mu \mathrm{m} .{ }^{*} \mathrm{P}<0.05$, ${ }^{* *} \mathrm{P}<0.01$, ${ }^{\star *} \mathrm{P}<0.001$, Data are presented as mean $\pm \operatorname{SD}(\mathrm{n} \geq 3)$. 

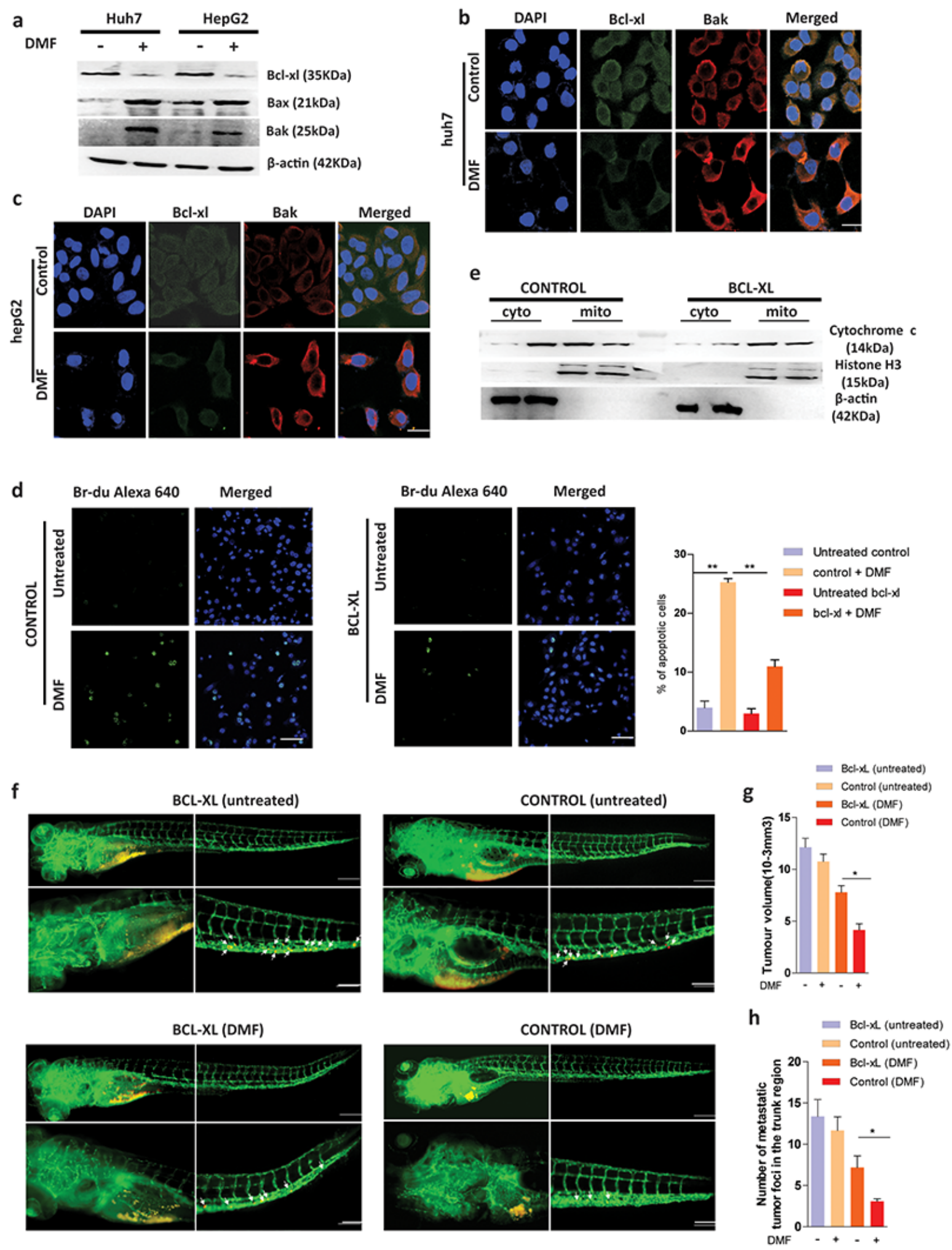

\section{Figure 5}

DMF's induced apoptosis and metastasis inhibition depends on Bcl-xL downregulation (a) Cells were treated with DMF or DMSO for 48hours, and then the expression of mitochondria apoptosis-related proteins was analyzed by western blotting. (b, c) Huh7 and HepG2 cells were treated with DMF for $48 \mathrm{H}$. The cells were fixed, and the gene expression was assessed by immunofluorescence assay. (d) Bcl-xl overexpression represses DMF-induced apoptosis in huh7 cells. Alexa Fluor® 640 Tunnel assay was used 
to assess apoptosis rate in GFP expressing cells treated with or without DMF for $48 \mathrm{H}$. (e) Bcl-xl overexpression suppresses DMF-induced increase in cytosolic cytochrome $\mathrm{c}$ in huh7 cells treated with or without DMF for $48 \mathrm{H}$ (f) Bcl-xl overexpression attenuates DMF-induced inhibition of tumour growth and metastasis. Representative images of the head and tail regions showing tumour cells and disseminated tumour foci in tumour-bearing zebrafish embryos treated DMF or vehicle control ( $n=10$ embryos/group). Arrowheads point to tumour cells. Scale bar $50 \mu \mathrm{m} .(\mathrm{g}, \mathrm{h})$ Quantification of the tumour volume and number of disseminated tumour foci in the trunk region and tumour volume. ${ }^{*} P<0.05,{ }^{*} P<0.01$. Data are presented as mean $\pm \operatorname{SD}(n \geq 3)$. 
a

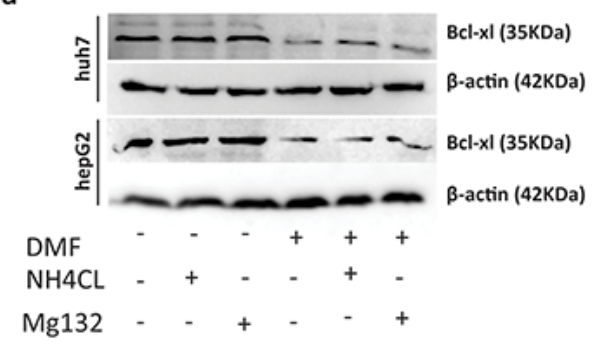

d

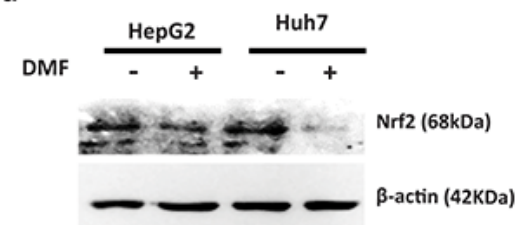

b

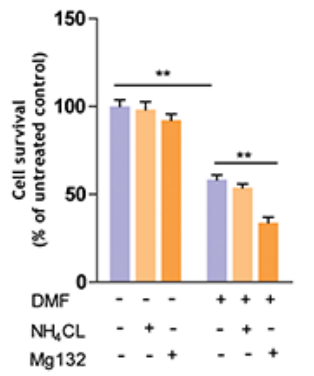

c

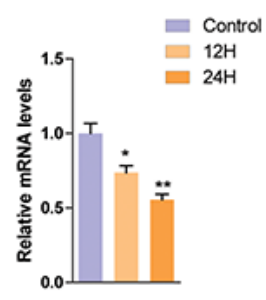

e
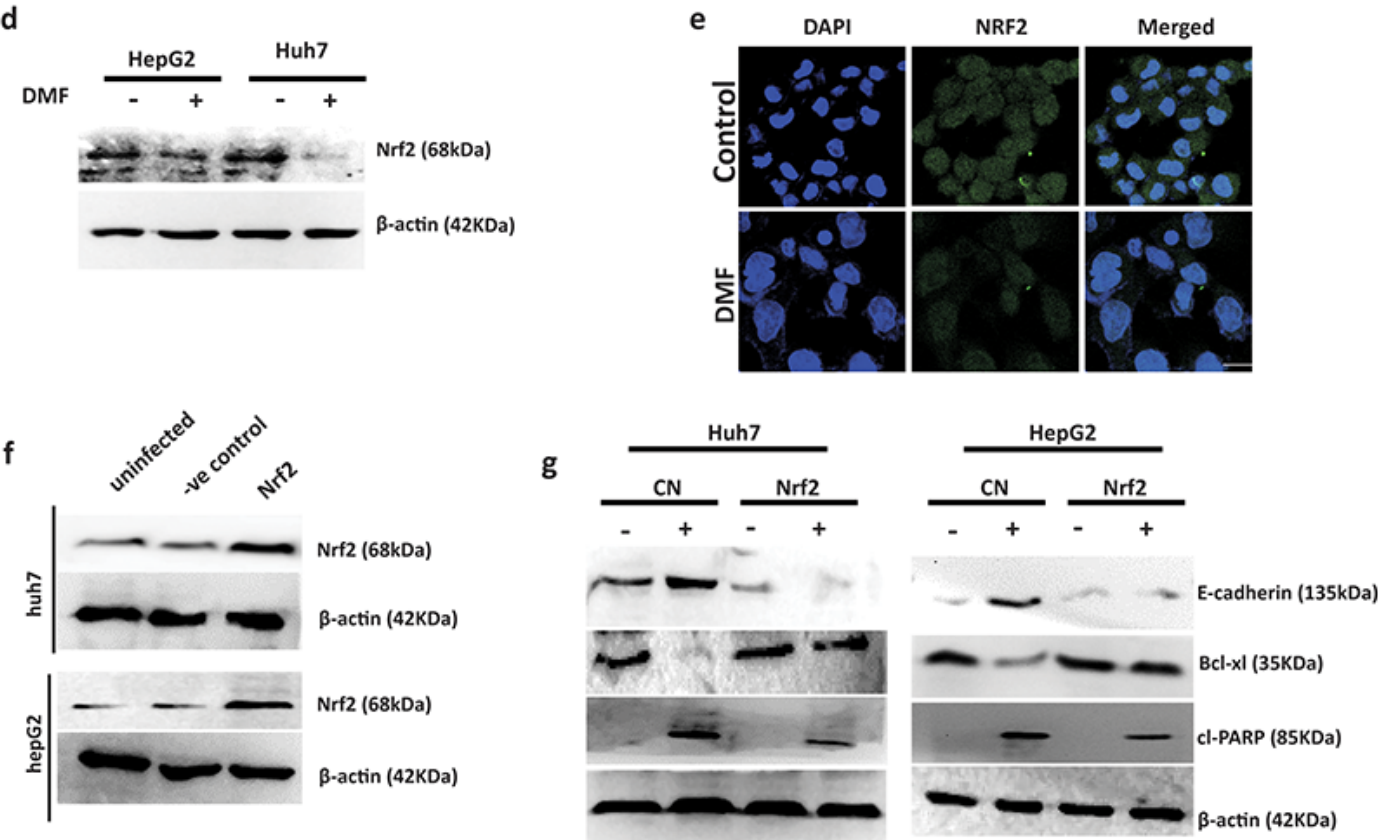

h
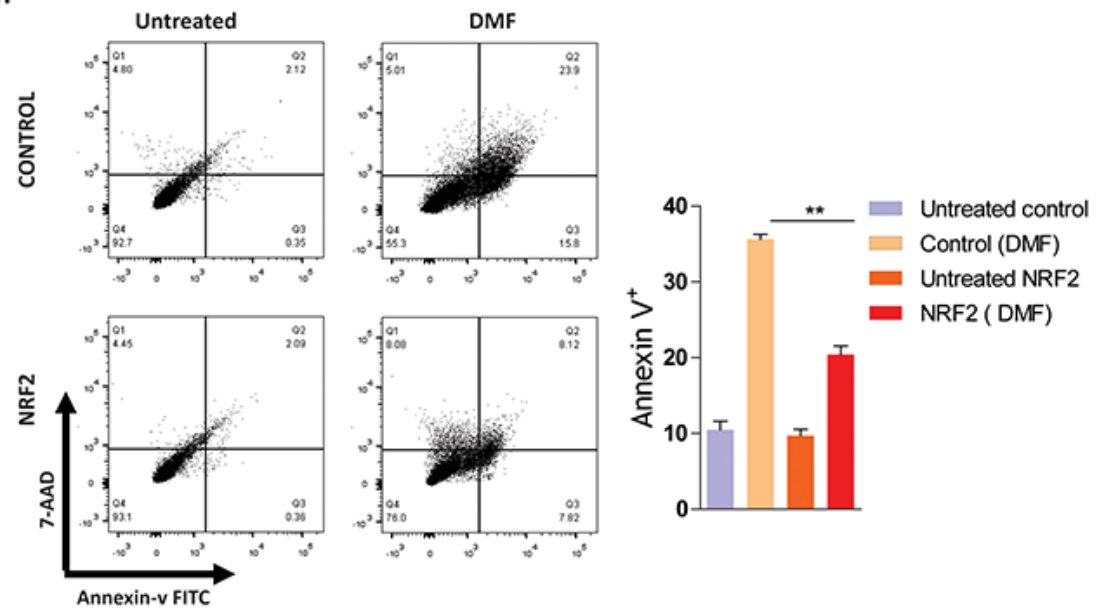

\section{Figure 6}

DMF downregulate Bcl-xL through NRF2 regulation (a) HCC cells were pre-treated with $\mathrm{NH} 4 \mathrm{Cl}(5 \mathrm{mM})$, MG132 $(10 \mu \mathrm{M})$ for $1 \mathrm{~h}$, followed by DMF treatment $(150 \mu \mathrm{M})$ for $24 \mathrm{~h}$ and the protein level of Bcl-xl was detected. (b) Cells were pre-treated with $\mathrm{NH} 4 \mathrm{Cl}(5 \mathrm{mM}), \mathrm{MG} 132(10 \mu \mathrm{M})$ for $1 \mathrm{~h}$, followed by DMF treatment $(150 \mu \mathrm{M})$ for $48 \mathrm{~h}$. Cell death was determined using CCK-8 assay. (c) Bcl-xl mRNA was extracted from huh7 and quantified using qRT-PCR after DMF treatment (d) Huh7 and hepG2 cells were treated with DMF 
for $48 \mathrm{H}$, and expression of Nrf2 was assessed using western blot. (e) Immunofluorescence staining for Nrf2 in DMF-treated huh7 cells (F) Nrf2 overexpression in huh7 and hepG2 cells (f) HCC cells were transfected with Nrf2 or control plasmid and subjected to immunoblot analysis (g) Nrf2 overexpression suppresses DMF-induced Bcl-xl and N-cadherin downregulation in huh7 and hepG2 cells. (h) Cells transfected with Nrf2 or control plasmid were treated with DMF for $48 \mathrm{H}$. Apoptosis rate was analyzed with 7-AAD/Annexin V-FITC stain using flow cytometry. Unpaired t-test was used for the statistical analysis. ${ }^{*} P<0.05, * * P<0.01$. Data are presented as mean $\pm \operatorname{SD}(n \geq 3)$.

$$
\text { a }
$$

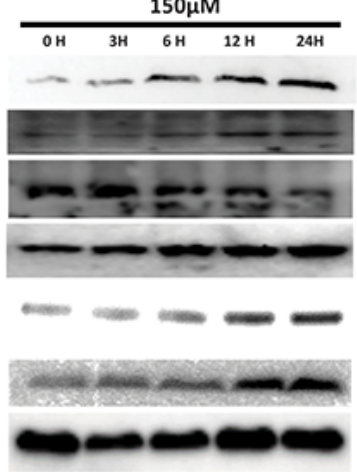

Huh7

buni $\frac{\text { Huh7 }}{-+} \frac{\text { HepG2 }}{-+}$

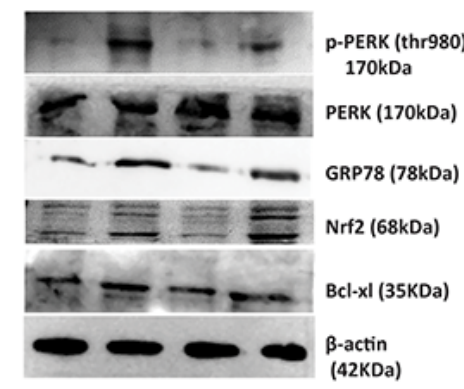

e

- Control

$\square 4 \mu 8 \mathrm{C}$

- GSK2606414

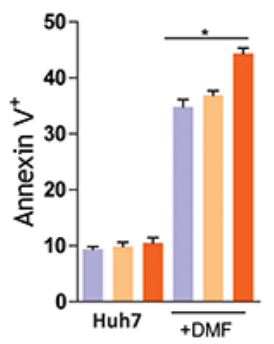

f Control

- $4 \mu 8 \mathrm{c}$

- GSK2606414

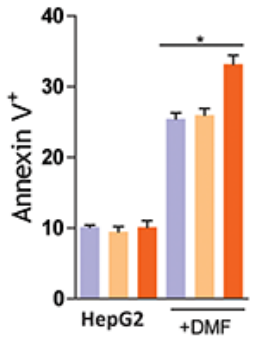

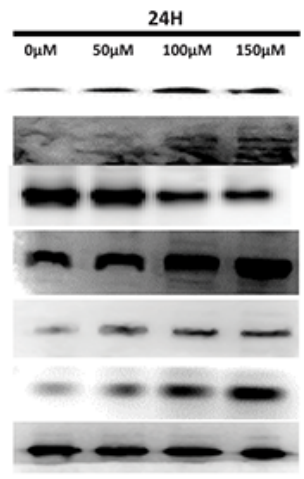

Huh7

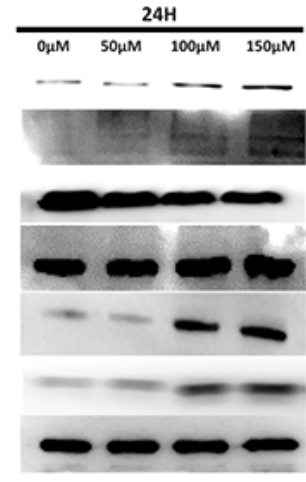

HepG2

c

- Control

- ER-stresss adapted

- Control (DMF)

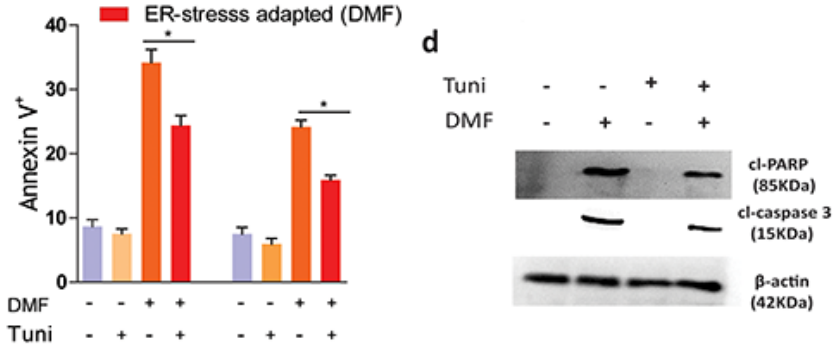

Huh7

HepG2

g

$\begin{array}{llll}\text { PERK } & \text {-ve } & \mathbf{2}\end{array}$
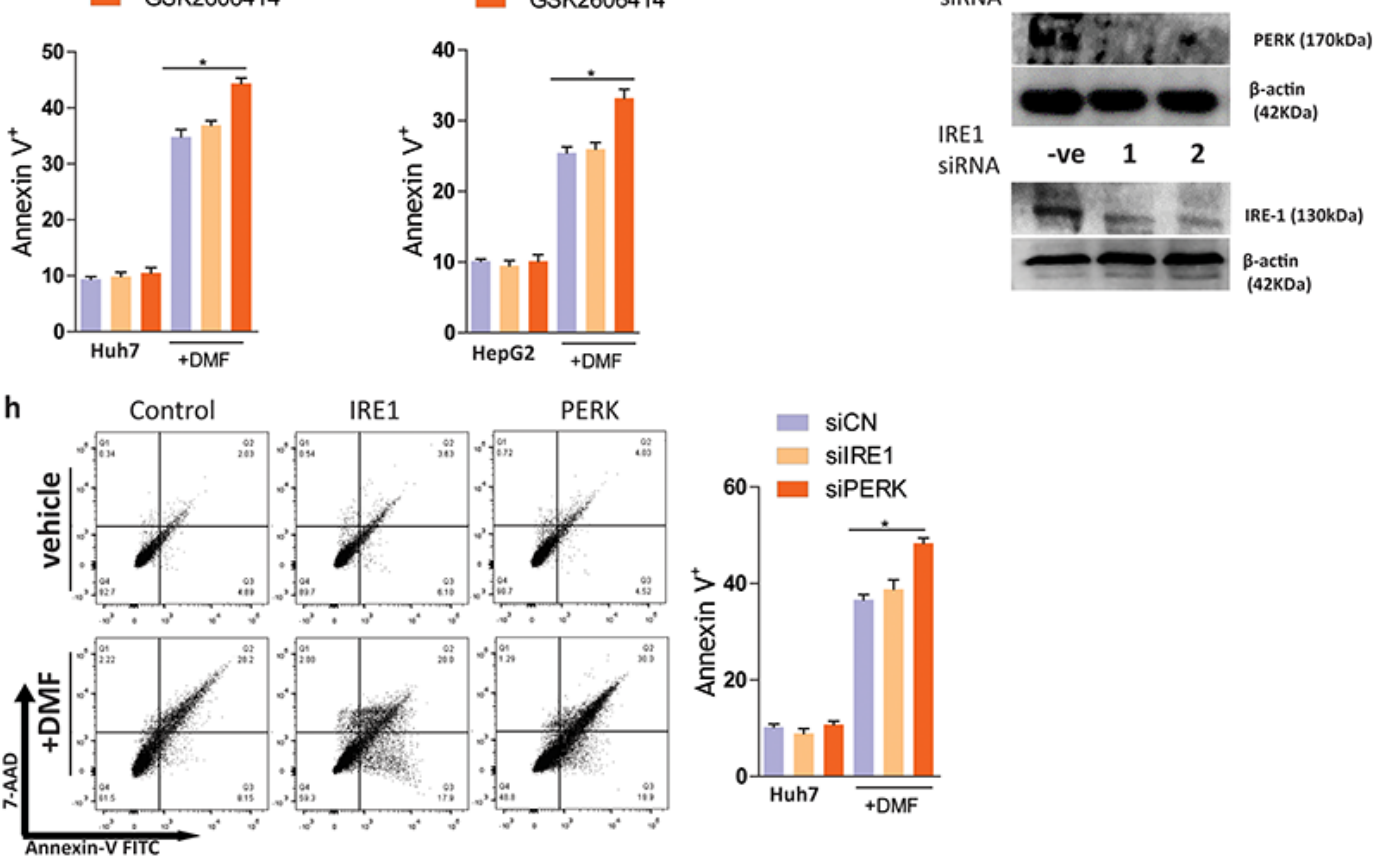


\section{Figure 7}

Endoplasmic reticulum (ER) stress protects from DMF-induced apoptosis (a) HCC cells were treated in a concentration or time-dependent manner, and expression of UPR/ER stress-related proteins including $\mathrm{p}$ PERK, p-eif2a, IRE1, CHOP, and GRP78 were detected by immunoblotting. (b) HCC cells were pretreatment of $4 \mu \mathrm{g} / \mathrm{mL}$ of tunicamycin for 4 days, and immunoblotting of relevant genes were performed. (c) HCC cells were pretreatment of $4 \mu \mathrm{g} / \mathrm{mL}$ of tunicamycin for 4 days were treated with DMF. Cell death was measured by Annexin V-FITC/7-AAD staining. (d) Western blotting analyses of cleaved caspase3 and cleaved PARP in DMF-treated huh7 cells pretreatment of $4 \mu \mathrm{g} / \mathrm{mL}$ tunicamycin for 4 days. (e, f) Huh7 and HepG2 cells were pre-treated with UPR inhibitors $4 \mu 8 \mathrm{C}$ (IRE1i, $5 \mu \mathrm{M})$ and GSK2606414 (PERKi, $10 \mu \mathrm{M})$ for 1 hour and then treated with or without DMF for $48 \mathrm{H}$. Cell death was measured by Annexin V-FITC/7-AAD staining (g) Huh7 cells were transfected with two siRNAs targeting IRE1 and PERK mRNAs. The knockdown efficacy was assessed using immunoblot analysis ( $h$ ) Cells transfected with IRE1, PERK or control siRNAs were treated with DMF for 48hours, and cell death was assessed using Annexin V-FITC/7-AAD staining. Unpaired t-test was used for the statistical analysis. ${ }^{*} P<.05$. Data are presented as mean \pm SD $(n \geq 3)$. 

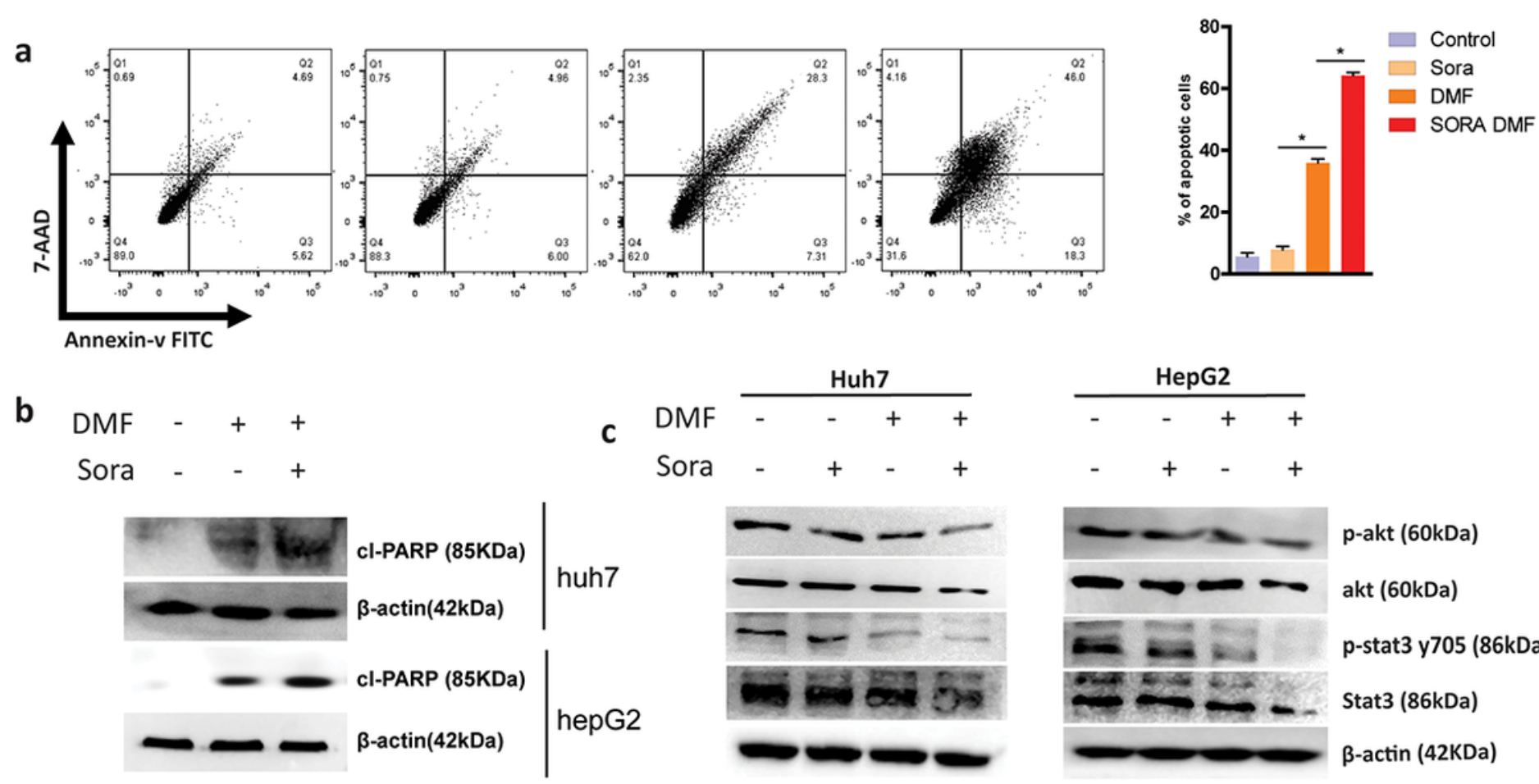

d
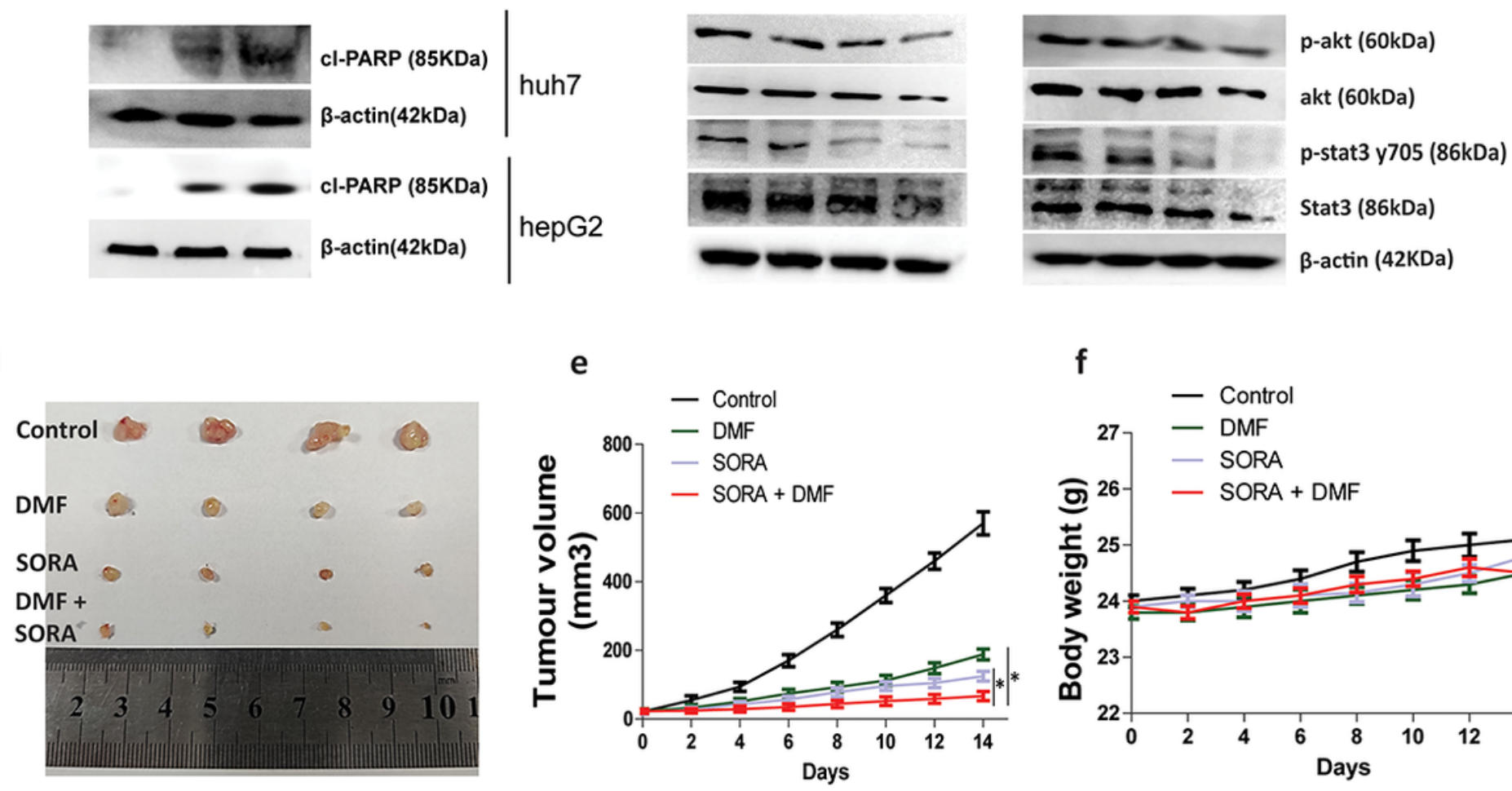

f

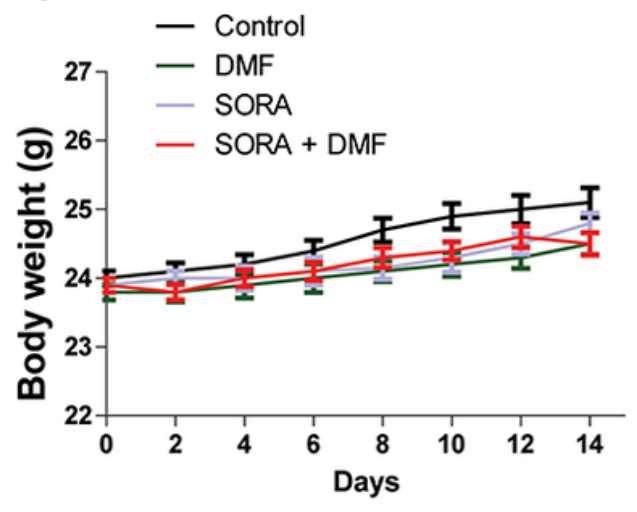

\section{Figure 8}

Dimethyl fumarate enhances the efficacy of sorafenib in vivo (a) Huh7 cells were treated with DMF and/ sorafenib for $48 \mathrm{H}$. Apoptosis rate was analyzed with Annexin V-FITC/7-AAD stain using flow cytometry. (b, c) Western blotting analyses of cleaved PARP, AKT, p-AKT, STAT3, p-STAT3. (d) Huh7 cells were injected into the animal to establish the xenograft model. Sorafenib and DMF were both dissolved in methylcellulose. Control group mice were treated with methylcellulose, and treatment group mice received DMF (30 mg/kg body weight i.p.), sorafenib (30 mg/kg/day, oral) or both daily for 14 days. (e, f) The volume and weight of the tumours in the two groups were recorded. Results are mean $\pm S D(n=5)$, **P $\leq$ 0.01 .

\section{Supplementary Files}


This is a list of supplementary files associated with this preprint. Click to download.

- SupplementaryMaterial2.pdf

- SupplementaryfiguresR3.pdf 